\title{
Pericyte-targeting prodrug overcomes tumor resistance to vascular disrupting agents
}

\author{
Minfeng Chen, ${ }^{1,2}$ Xueping Lei, ${ }^{1,2}$ Changzheng Shi, ${ }^{3}$ Maohua Huang, ${ }^{1,2}$ Xiaobo Li, ${ }^{1,2}$ Baojian Wu,,${ }^{1,2}$ Zhengqiu Li, ${ }^{1,2}$ Weili Han, ${ }^{4}$ Bin Du, ${ }^{3}$ \\ Jianyang Hu, ${ }^{1,2}$ Qiulin Nie, ${ }^{1,2}$ Weiqian Mai, ${ }^{1,2}$ Nan Ma, ${ }^{1,2}$ Nanhui Xu, ${ }^{1,2}$ Xinyi Zhang, ${ }^{1,2}$ Chunlin Fan, ${ }^{1,2}$ Aihua Hong, ${ }^{5}$ Minghan Xia, ${ }^{3}$ \\ Liangping Luo, ${ }^{3}$ Ande Ma, ${ }^{4}$ Hongsheng Li, ${ }^{6}$ Qiang Yu, ${ }^{7}$ Heru Chen, ${ }^{1,2}$ Dongmei Zhang ${ }^{1,2}$ and Wencai Ye ${ }^{1,2}$ \\ 'College of Pharmacy, and ${ }^{2}$ Guangdong Province Key Laboratory of Pharmacodynamic Constituents of Traditional Chinese Medicine and New Drugs Research, Jinan University, Guangzhou, China. ${ }^{3}$ The First \\ Affiliated Hospital of Jinan University, Guangzhou, China. ${ }^{4}$ School of Public Health and Tropical Medicine, Southern Medical University, Guangzhou, China. ${ }^{5}$ Analytical and Testing Center, Jinan University, \\ Guangzhou, China. ${ }^{6}$ Cancer Center of Guangzhou Medical University, Guangzhou, China. Department of Physiology, Yong Loo Lin School of Medicine, National University of Singapore, Singapore.
}

\begin{abstract}
Blood vessels in the tumor periphery have high pericyte coverage and are resistant to vascular disrupting agents (VDAs). VDA treatment resistance leads to a viable peripheral tumor rim that contributes to treatment failure and disease recurrence. Here, we provide evidence to support a hypothesis that shifting the target of VDAs from tumor vessel endothelial cells to pericytes disrupts tumor peripheral vessels and the viable rim, circumventing VDA treatment resistance. Through chemical engineering, we developed Z-GP-DAVLBH (from the tubulin-binding VDA desacetylvinblastine monohydrazide [DAVLBH]) as a prodrug that can be selectively activated by fibroblast activation protein $\alpha$ (FAP $\alpha$ ) in tumor pericytes. Z-GP-DAVLBH selectively destroys the cytoskeleton of $\mathrm{FAP} \alpha$-expressing tumor pericytes, disrupting blood vessels both within the core and around the periphery of tumors. As a result, Z-CP-DAVLBH treatment eradicated the otherwise VDA-resistant tumor rim and led to complete regression of tumors in multiple lines of xenografts without producing the drug-related toxicity that is associated with similar doses of DAVLBH. This study demonstrates that targeting tumor pericytes with an FAP $\alpha$-activated VDA prodrug represents a potential vascular disruption strategy in overcoming tumor resistance to VDA treatments.
\end{abstract}

\section{Introduction}

Vascular disrupting agents (VDAs), mainly comprising tubulinbinding compounds, act primarily on tumor vascular endothelial cells (ECs) and alter the cellular tubulin cytoskeleton. Ultimately, VDAs selectively destroy the well-established tumor vasculature to cause acute shutdown of blood flow, eliciting extensive necrosis in the tumor core (1). However, the tumor periphery seems to be resistant to VDAs, as evidenced by the presence of a viable rim, which eventually leads to rapid regrowth of the tumor after a single VDA treatment and hampers the efficacy and clinical drug development of VDAs $(1,2)$. Although combination strategies with antiangiogenic agents, cytotoxic drugs, or radiotherapy have been applied in preclinical and clinical trials (3), they also failed to eliminate the viable rim in the tumor periphery, indicating the necessity of developing VDAs from a new perspective.

Several studies showed that blood vessels in the tumor periphery, which have high pericyte coverage, are less responsive to VDAs, indicating that pericytes might be closely related to VDA treatment resistance (4-7). Pericytes play an important role in promoting microvasculature stabilization (8), and genetic ablation of pericytes gives rise to vessel leakage and hemorrhage $(9,10)$. Therefore, we propose a new therapeutic strategy to eliminate the

Authorship note: M. Chen and X. Lei are co-first authors.

Conflict of interest: The authors have declared that no conflict of interest exists.

Submitted: March 31, 2017; Accepted: July 11, 2017.

Reference information: / Clin Invest. 2017;127(10):3689-3701.

https://doi.org/10.1172/JCI94258. tumor rim and overcome the inherent defect of VDAs by transforming the VDA target from tumor ECs to pericytes.

The enzyme-activated prodrug strategy is effective in enhancing the selectivity of parent drugs in enzyme-expressing cells. Fibroblast activation protein $\alpha$ (FAP $\alpha$ ), a type II integral membrane serine protease, belongs to the dipeptidyl peptidase (DPP) subfamily that typically cleaves peptide substrates after a proline residue (11). The closest homolog of FAP $\alpha$ is DPP-IV, which shares $48 \%$ amino acid similarity (12). However, unlike DPP-IV, which is widely expressed in human tissues, FAP $\alpha$ is specifically overexpressed in cancer-associated fibroblasts (CAFs) and pericytes in more than $90 \%$ of malignant human epithelial cancers but is virtually undetectable in most normal adult tissues $(13,14)$. In addition, in contrast to other serine proteases of the DPP subfamily, $\mathrm{FAP} \alpha$ also possesses endopeptidase activity that can specifically cleave N-terminal benzyloxy carbonyl-blocked (Z-blocked) GlyPro (Z-GP) dipeptide-linked substrates (15). The distinct dipeptide substrate hydrolytic activity of FAP $\alpha$ and its restricted expression in the tumor microenvironment make FAP $\alpha$ an ideal target for an enzyme-activated prodrug strategy that shifts the VDA target from tumor ECs to pericytes or CAFs.

Recently, several FAP $\alpha$-activated prodrugs have been developed and demonstrated to target CAFs to inhibit tumor growth $(13,14,16)$. To construct an FAP $\alpha$-activated prodrug that specifically targets tumor pericytes, we screened a panel of known VDA drugs, including combretastatins, vinca alkaloids, and their derivatives, for drugs with higher sensitivity toward pericytes than fibroblasts. Through this approach, desacetylvinblastine 
monohydrazide (DAVLBH), a derivative of vinblastine (17), was identified and validated as showing the highest selectivity toward pericytes over fibroblasts. Therefore, DAVLBH was chosen as the parent drug to be coupled with Z-GP to produce an FAP $\alpha$-activated prodrug, Z-GP-DAVLBH. Here, we show that Z-GP-DAVLBH disrupts blood vessels both in the core and in the periphery of tumors by selectively targeting FAP $\alpha$-expressing tumor pericytes. This results in complete regression of multiple lines of tumor xenografts without a viable rim. To the best of our knowledge, this is the first report of disrupting tumor vessels by transforming the VDA target from tumor ECs to pericytes, through which it overcomes VDA treatment resistance.

\section{Results}

Pericyte-covered vessels are insensitive to DAVLBH. We first investigated whether blood vessels in the tumor periphery are resistant to DAVLBH. Consistent with previous reports (7), blood vessels in the periphery of MDA-MB-231 tumor xenograft are covered with more FAP $\alpha$-expressing pericytes than the tumor core (Supplemental Figure 1; supplemental material available online with this article; https://doi.org/10.1172/JCI94258DS1). Using double staining of FAP $\alpha$ and CD31 (a marker of ECs) in MDA-MB-231 tumor xenografts, we found that DAVLBH $(2.0 \mu \mathrm{mol} / \mathrm{kg})$ caused almost complete loss of CD31-staining ECs in the tumor core while having negligible effects on the vessels in the tumor periphery within 4 hours of treatment (Figure 1A). These results were confirmed by transmission electron microscopy, in which DAVLBH $(2.0 \mu \mathrm{mol} / \mathrm{kg})$ induced EC rounding and blebbing after 2 hours of treatment, followed by focal loss of EC and exposure of basal lamina in the tumor core vessels after 4 hours of treatment, when the pericytes remained adherent to the lamina. However, no significant change was observed in ECs or pericytes of vessels in the tumor rim (Figure 1B). As a result, tumors treated with DAVLBH for 2 days displayed extensive necrosis in the core, but had an obvious viable rim in the periphery, which was indicated by a Ki67 proliferation index equal to that in the vehicle group (Figure 1C). Subsequently, a pericyte-EC-cocultured system was established to mimic tumor vessels. In accordance with the in vivo effects, DAVLBH (500 nM) disrupted the preestablished HUVEC tubes within 1 hour, while it showed negligible effects on the HUVEC tubes cocultured with pericytes (Figure 1D). These data are consistent with the effects of classical VDAs, as reported previously (4-7), which showed that VDAs are insensitive to vessels with high pericyte coverage, further supporting the idea that pericytes may contribute to VDA resistance.

$Z$-GP-DAVLBH selectively disrupts the pericyte cytoskeleton in an FAP $\alpha$-dependent manner. We next sought to investigate whether our newly synthesized FAP $\alpha$-activated VDA prodrug can shift the target of DAVLBH from tumor ECs to pericytes. First, we evaluated the proof-of-concept of Z-GP-DAVLBH as a prodrug (Figure 2A). The results showed that Z-GP-DAVLBH was specifically hydrolyzed by recombinant human FAP $\alpha(\operatorname{rhFAP} \alpha)$, and the catalytic efficiency $\left(K_{c a t} / K_{m}\right)$ of rhFAP $\alpha$ for Z-GP-DAVLBH was $2483 \mathrm{M}^{-1} \mathrm{~s}^{-1}$ (Figure 2B). However, Z-GP-DAVLBH was resistant to hydrolysis by DPP-IV, the closest homolog of FAP $\alpha$ (Supplemental Figure 2). Interestingly, we found that prolyl endopeptidase (PEP), a cytosolic prolyl oligopeptidase that possesses FAP $\alpha$-like endopeptidase activity (18), showed approximately $1 / 7$ of the hydrolytic activity on Z-GP-DAVLBH compared with FAP $\alpha$ (Supplemental Figure 2). Previous researches have demonstrated that the conserved active site residues Arg123, Glu203, Glu204, Tyr656, and Asn704 are essential for the endopeptidase activity of FAP $\alpha$ (Protein Data Bank 1Z68) $(15,19,20)$. We therefore constructed 5 mutant FAP $\alpha$ plasmids (R123A, E203A, E204A, Y656F, and N704A) to investigate whether Z-GP-DAVLBH can be specifically cleaved by FAP $\alpha$. The WT and mutant FAP $\alpha$ plasmids were transfected into HEK293T cells to establish HEK-293T ${ }^{\text {FAPa-WT }}$, HEK-293T ${ }^{\text {R123A }}$, HEK293T ${ }^{\mathrm{E} 203 \mathrm{~A}}$, HEK-293T ${ }^{\mathrm{E} 204 \mathrm{~A}}$, HEK-293T ${ }^{\mathrm{Y} 656 \mathrm{~F}}$, and HEK-293T ${ }^{\mathrm{N} 704 \mathrm{~A}}$ cells. We found that Z-GP-DAVLBH was significantly hydrolyzed in HEK-293T cells transfected with the FAP $\alpha$ WT plasmid and that the hydrolysis ability was dramatically reduced by FAP $\alpha$ mutants (Figure 2C and Supplemental Figure 3A). In addition, we found that HEK-293T cells transfected with the WT or mutant FAP $\alpha$ plasmids pretreated with $100 \mu \mathrm{M}$ of ValboroPro, the nonselective inhibitor of DPPs (including FAP $\alpha$ ) (21), showed negligible hydrolysis toward Z-GP-DAVLBH (Supplemental Figure 3B). Furthermore, we found that Z-GP-DAVLBH could be promptly and effectively activated in FAP $\alpha$-overexpressing $\left(\mathrm{FAP}^{+}\right) \mathrm{MDA}-\mathrm{MB}-231$ tumors (Figure 2D). These results demonstrate that Z-GP-DAVLBH can be selectively activated by FAP $\alpha$.

To determine whether Z-GP-DAVLBH could be activated by tumor pericytes and consequently destroy cellular cytoskeleton, human brain vascular pericytes (HBVPs) were transfected with FAP $\alpha$ WT plasmid to generate HBVP ${ }^{\mathrm{FAP} \alpha-\mathrm{WT}}$, mimicking tumor pericytes. We showed that approximately $80 \%$ of Z-GP-DAVLBH incubated with HBVP ${ }^{\text {FAPa-WT }}$ was hydrolyzed to DAVLBH. However, the non-FAP $\alpha$-expressing (FAP $\left.\alpha^{-}\right)$cells, including HBVPs, HUVECs, and MDA-MB-231 cells, showed no hydrolysis of Z-GP-DAVLBH (Figure 2E). As a prodrug, Z-GP-DAVLBH should exhibit reduced microtubule depolymerization effect compared with the active drug DAVLBH. Our in vitro experimental data show that this is indeed the case. DAVLBH significantly inhibited tubulin polymerization, whereas the activity of Z-GP-DAVLBH was greatly diminished (Figure $2 \mathrm{~F}$ ). Therefore, it is tempting to speculate that the microtubule-depolymerizing and cytoskeleton-destroying effect of Z-GP-DAVLBH would only occur after it is hydrolyzed by FAP $\alpha$ to become DAVLBH in $\mathrm{FAP}^{+}$pericytes. As expected, contraction of $\beta$-tubulin and $\mathrm{F}$ actin cytoskeletons of $\mathrm{HBVP}^{\mathrm{FAPa}-\mathrm{WT}}$ cells was observed following incubation with $2.5 \mathrm{nM} \mathrm{Z}$-GP-DAVLBH for 30 minutes (Figure $2 \mathrm{G})$. In contrast, the cytoskeleton of FAP $\alpha^{-}$cells with the same treatment showed negligible changes (Figure $2 \mathrm{G}$ ).

$Z-G P-D A V L B H$ disrupts tumor vessels by selectively targeting FAP $\alpha$-expressingpericytes. Next, we examined whetherZ-GP-DAVLBH disrupts tumor vessels by preferentially targeting $\mathrm{FAP}^{+}$pericytes. Our results showed that Z-GP-DAVLBH (500 nM), but not DAVLBH, initially caused shrinkage of the cytoskeleton of HBVP ${ }^{\mathrm{FAPa}-\mathrm{WT}}$ cells attached to HUVEC tubes within 30 minutes (Figure 3A and Supplemental Figure 4). We postulated that the selective effect of Z-GP-DAVLBH on pericytes in the cocultured system could be due to the accumulation of drugs in HBVP ${ }^{\mathrm{FAP}-\mathrm{WT}}$ cells. To test this hypothesis, we developed a fluorescent-conjugated agent, Z-GP-DAVLBHBODIPY, to directly analyze the distribution of Z-GP-DAVLBH in

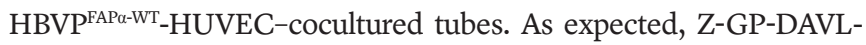
BH-BODIPY selectively accumulated in HBVP ${ }^{\text {FAPa-WT }}$ cell coverings 
A
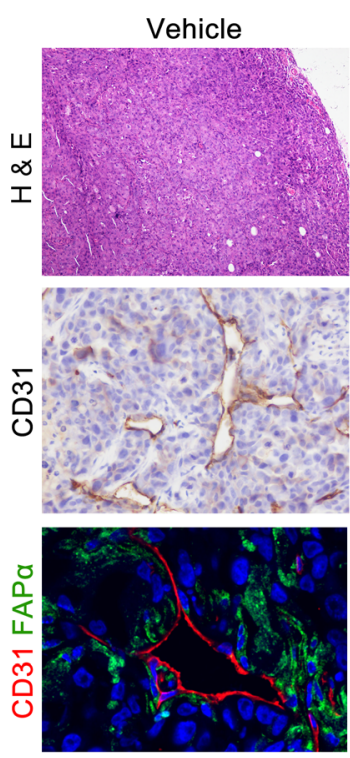

B
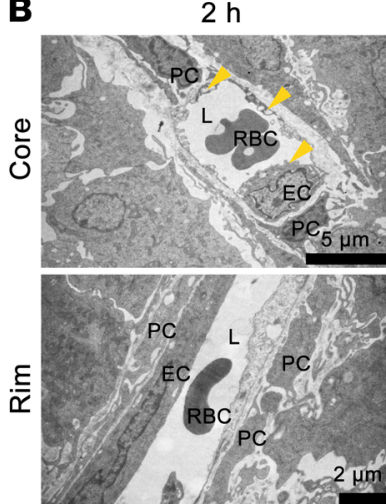

DAVLBH
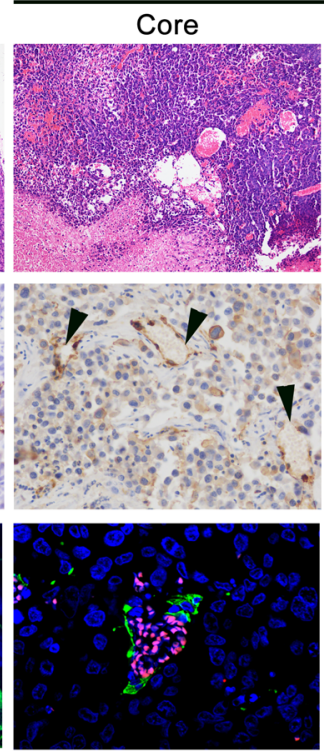
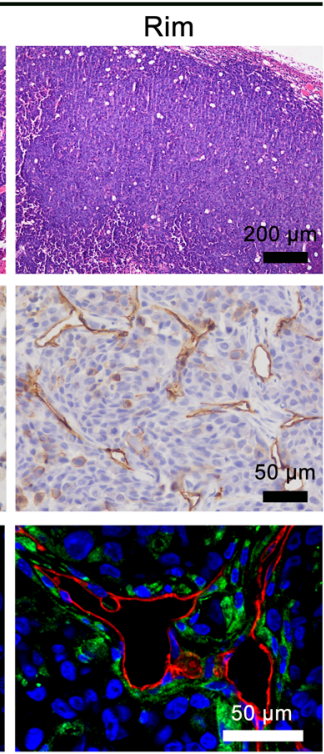

C
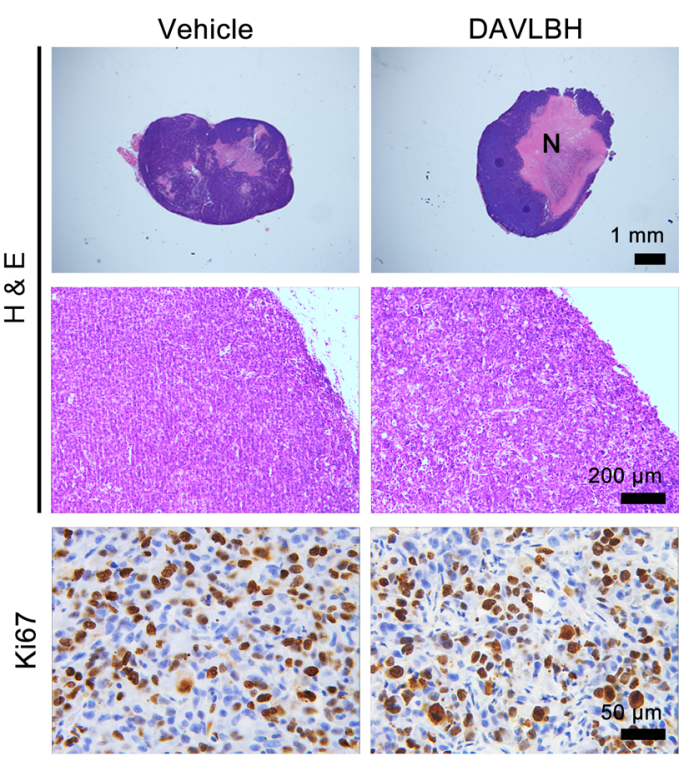

HUVEC/HBVPNC

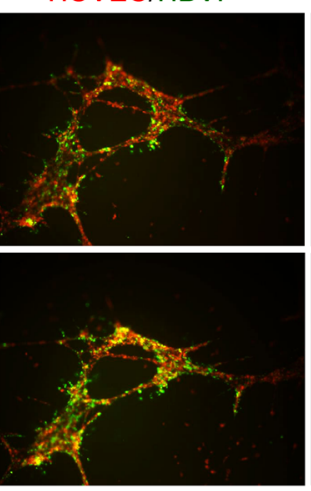

HUVEC/HBVPFAPa-WT

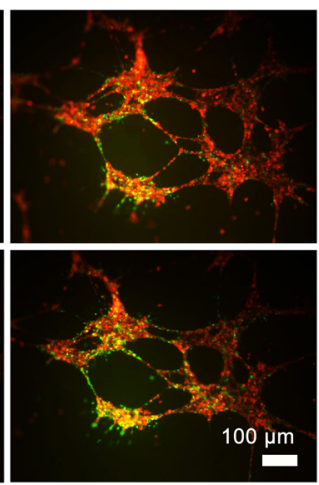

Figure 1. Vessels in the tumor periphery with higher pericyte coverage show resistance to DAVLBH. For in vivo experiments, mice bearing MDA-MB-231 xenografts received i.v. injection of $2 \mu \mathrm{mol} / \mathrm{kg}$ DAVLBH, and the tumors were harvested 2 hours, 4 hours, or 2 days later. (A) H\&E, CD31, and FAP $\alpha / C D 31$ staining show a marked vascular disruption in tumor core, but not in peripheral tumor, after 4 hours of treatment $(n=5)$. The black arrowheads indicate the loss of ECs, and autofluorescent rbc appear as pink points in FAB $\alpha / C D 31$-stained sections. Top row scale bars: $200 \mu \mathrm{m}$. Middle row scale bars: $50 \mu \mathrm{m}$. Bottom row scale bars: $50 \mu \mathrm{m}$. (B) The transmission electron microscope images show the effects of DAVLBH on tumor vessels. The yellow arrowheads indicate EC blebbing, and the blue arrowheads indicate the loss of ECs. L, lumen; PC, pericyte. Top row scale bars: $5 \mu \mathrm{m}$. Bottom row left scale bar: $2 \mu \mathrm{m}$. Bottom row right scale bar: $10 \mu \mathrm{m}$. (C) H\&E staining shows extensive necrosis Top row scale bars: $1 \mathrm{~mm}$. Middle row scale bars: $200 \mu \mathrm{m}$. Bottom row scale bars: $50 \mu \mathrm{m}$. (N) in tumor core after 2 days of treatment; Ki67 staining shows similar proliferation between 2 groups $(n=5)$. (D) The EC- (red) and pericytecocultured (green) systems show DAVLBH selectively damages the HUVEC tubes (white arrows), but has negligible effect on the HBVPNC-HUVEC and HBVPFAPa-WT-HUVEC-cocultured tubes $(n=3)$. Scale bars: $100 \mu \mathrm{m}$.

on the HUVEC tubes (Figure 3B). However, Z-GP-DAVLBH-BODIPY showed nonselective accumulation in both cells of the HBVPHUVEC-cocultured tubes (Supplemental Figure 5), indicating that the accumulation of drug in HBVP ${ }^{\mathrm{FAP} \alpha-W T}$ cells depends on FAP $\alpha$. As a result, Z-GP-DAVLBH selectively disrupted the HBVPFAP-WT HUVEC-cocultured tubes rather than HUVEC tubes or HBVP ${ }^{\mathrm{NC}}$ HUVEC tubes (Figure 3C). Immunohistochemical staining of CD31 and immunofluorescent double-staining of FAP $\alpha / C D 31$ in MDAMB-231 tumor tissues showed that Z-GP-DAVLBH $(2.0 \mu \mathrm{mol} / \mathrm{kg})$ treatment for 4 hours induced $\mathrm{FAP}^{+}$tumor pericyte retraction and detachment, producing a "hole-like" vessel disruption in both the tumor core and the periphery (Figure 4A). Transmission electron microscopy results confirmed that Z-GP-DAVLBH treatment for 1 hour induced pericyte shrinkage and detachment from the EC wall without affecting the ECs and disrupted the continuity of the EC linearity in the peripheral tumorvessels after 4 hours of treatment (Figure $4 \mathrm{~B})$. Taken together, these results demonstrate that Z-GP-DAVLBH disrupts tumor vessels by selectively targeting tumor pericytes.

To investigate whether Z-GP-DAVLBH disrupts the tumor vasculature in an FAP $\alpha$-dependent manner, we established a negative control compound, $t$-butyloxycarboryl-Ala-Pro-DAVLBH (Boc-AP-DAVLBH) (Supplemental Figure 6A), which was demonstrated to be completely resistant to rhFAP $\alpha-$, DPP-IV-, or PEP-mediated hydrolysis (Supplemental Figure 2). We found that Boc-AP-DAVLBH had a negligible effect on the cytoskeleton of HVBP ${ }^{\text {FAP-WT }}$, the morphology of HBVP ${ }^{\text {FAP }}-\mathrm{WT}-H U V E C-$ cocultured tubes, and tumor vessels in MDA-MB-231 xenografts (Supplemental Figure 6, B-D). These data demonstrate that FAP $\alpha$ acti- 
A

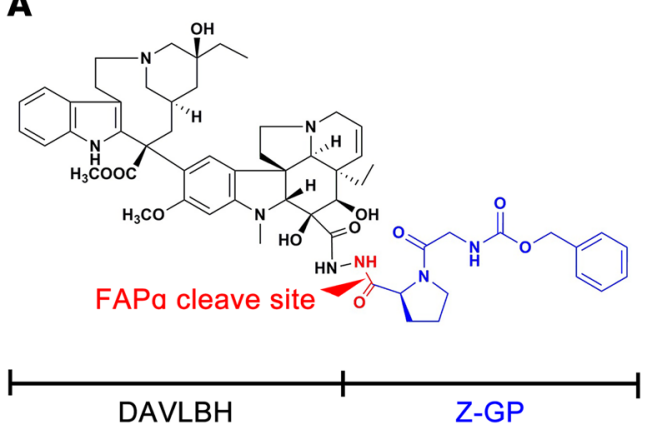

$\mathbf{C}_{100}$

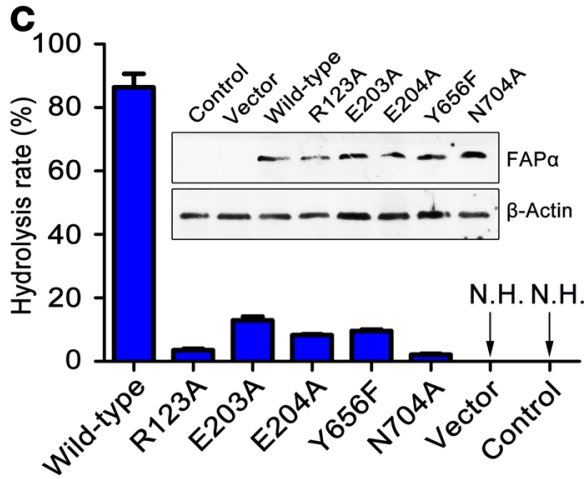

E

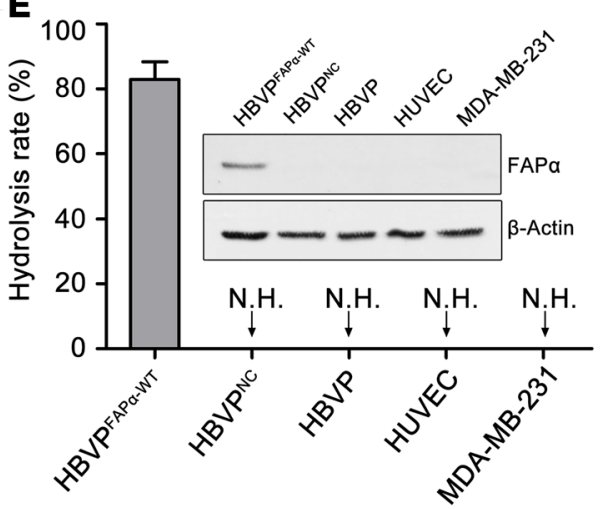

B

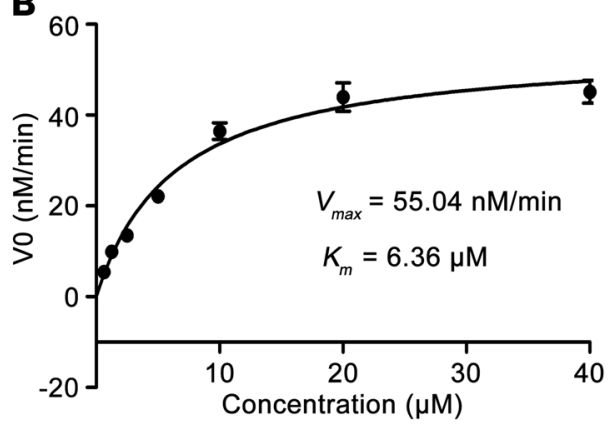

D

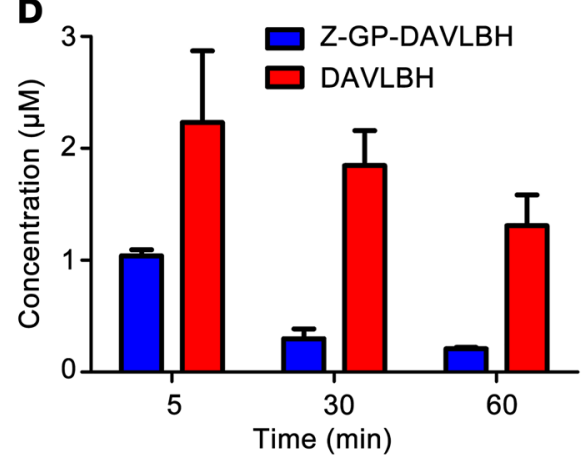

$\mathbf{F}$

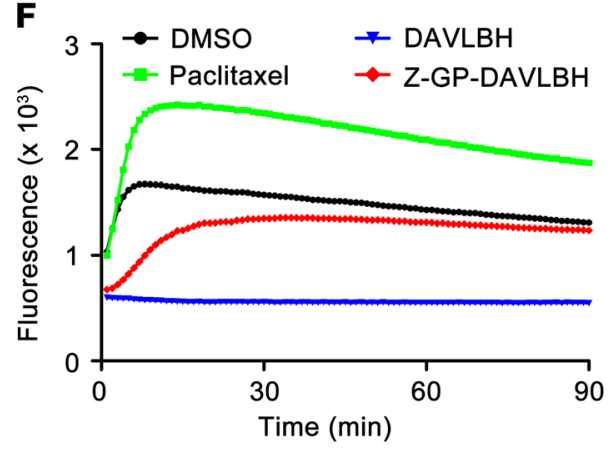

G

HBVPFAPa-WT
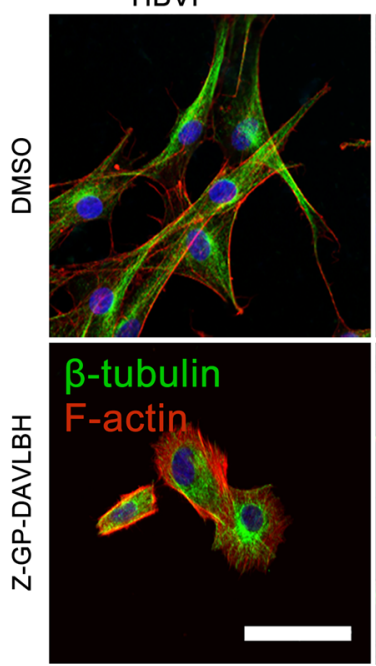

HBVPNC

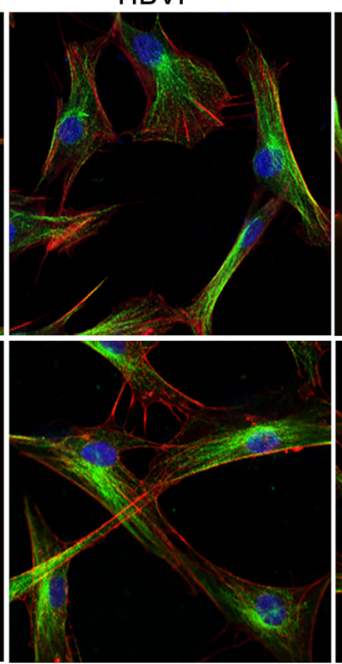

HBVP

HUVEC

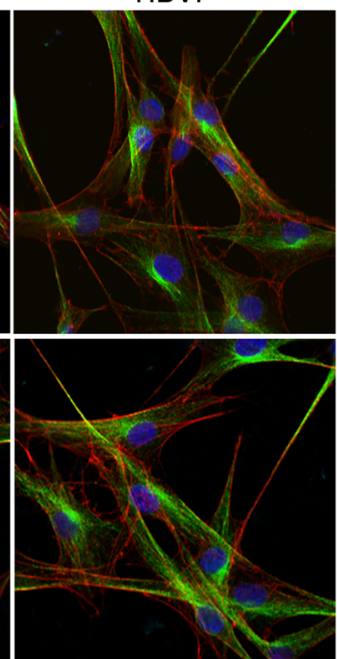

MDA-MB-231

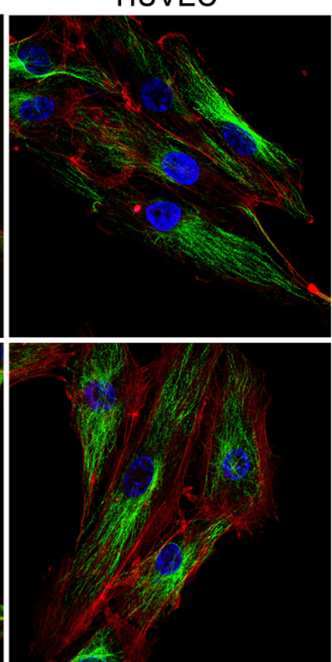

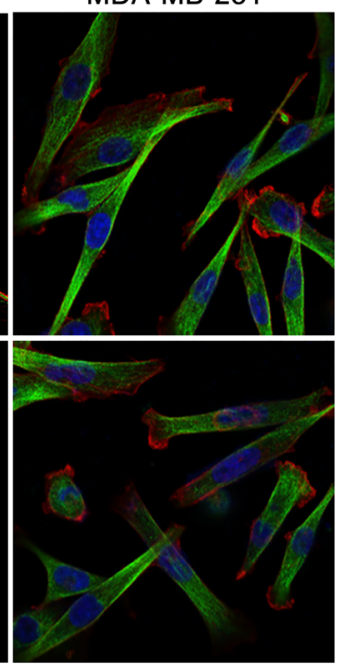


Figure 2. Z-GP-DAVLBH is selectively hydrolyzed by FAP $\alpha$ to release DAVL$\mathrm{BH}$ to disrupt the cytoskeleton of FAP $\alpha$-expressing pericytes. (A) Structure of Z-GP-DAVLBH. (B) Evaluation of the enzymatic kinetics of rhFAP $\alpha$ on Z-GP-DAVLBH. The substrate-velocity curve for cleavage of Z-CP-DAVLBH by rhFAP $\alpha(5 \mathrm{ng} / \mathrm{ml})$ is shown $(n=3)$. (C) Enzymatic efficacy of engineered FAP $\alpha$-expressing cells on Z-CP-DAVLBH. HEK-293T cells were transiently transfected with vector, WT FAP $\alpha$, or mutant FAP $\alpha$ plasmids (R123A, E203A, E204A, Y656F, N704A). Z-CP-DAVLBH $(10 \mu \mathrm{M})$ was cocultured with cells at $37^{\circ} \mathrm{C}$ for 2 hours, and hydrolysis was analyzed by LC/MS. Quantification of the hydrolysis rate is shown $(n=3)$. N.H., no hydrolysis. (D) Evaluation of the hydrolysis for Z-CP-DAVLBH in MDA-MB-231 tumor xenografts $(n=5)$. The concentrations of Z-GP-DAVLBH and DAVLBH in tumors were detected at 5, 30, and 60 minutes after i.v. injection of Z-CP-DAVLBH $(2.0 \mu \mathrm{mol} / \mathrm{kg})$. (E) Enzymatic ability of HBVPFAPa-WT, HBVPNC, HBVPs, HUVECs, and MDA-MB-231 against Z-CP-DAVLBH. Quantification of the hydrolysis rate is shown $(n=3)$. (F) Inhibition of tubulin polymerization by DAVLBH and Z-CP-DAVLBH in vitro. Purified porcine brain tubulin was incubated with the tested compounds at $1 \mu \mathrm{M}$. Effects on tubulin polymerization were monitored by fluorescence value measurement, with excitation at $360 \mathrm{~nm}$ and emission at $420 \mathrm{~nm}$ every 1 minute for 90 minutes at $37^{\circ} \mathrm{C}$. Paclitaxel $(3 \mu \mathrm{M})$ was used as positive control agent. (G) The effect of Z-GP-DAVLBH on the $\beta$-tubulin cytoskeleton of HBVPFAPa-WT, HBVP ${ }^{N C}$, HBVPs, HUVECs, and MDA-MB-231 cells $(n=3)$. The cells were treated with Z-GP-DAVLBH $(2.5 \mathrm{nM})$ for 30 minutes, and $\beta$-tubulin (green) and $\mathrm{F}$-actin (red) were observed with a confocal microscope. Data are shown as mean \pm SEM. Scale bar: $50 \mu \mathrm{m}$.

vation is essential for the in vitro and in vivo vascular disruption effects of Z-GP-DAVLBH.

Moreover, we found that Z-GP-DAVLBH was potently hydrolyzed in BALB-3T3 ${ }^{\text {FAPa-WT }}$ cells mimicking CAFs (Supplemental Figure 7A). However, Z-GP-DAVLBH, at concentrations up to $25 \mathrm{nM}$, had little effect on the cytoskeleton of BALB/3T3 ${ }^{\text {FAPa-WT }}$ cells or on FAP $\alpha$-positive CAFs in vivo within 6 days (Supplemental Figure 7, B and C). These data indicate that Z-GP-DAVLBH selectively targets tumor pericytes rather than CAFs.

Z-GP-DAVLBH selectively induces tumor vascular disruption in both the tumor core and the rim, leading to the elimination of the viable rim. To further monitor the vascular-disrupting and antitumor effects of Z-GP-DAVLBH, we conducted dynamic contrast-enhanced MRI (DCE-MRI) and diffusion-weighted MRI (DW-MRI) experiments on MDA-MB-231 tumor xenografts before and after Z-GP-DAVLBH (2.0 $\mu \mathrm{mol} / \mathrm{kg}$ ) administration. Our results showed that Z-GP-DAVLBH induced a similar reduction of $K^{\text {trans }}$ values, a constant that is directly proportional to the tumor vascular perfusion and permeability (22), as determined by DCE-MRI, in both the tumor core and the periphery (Figure 5A). Notably, unlike classical VDA, whose $K^{\text {trans }}$ values in the tumor rim recovered to nearly baseline levels at 24 to 48 hours after treatment $(23,24)$, Z-GP-DAVLBH reduced $K^{\text {trans }}$ values to levels consistently lower than baseline at a time period ranging from 4 hours to 18 days (Figure 5A). This finding may result from the reduced perfusion and decrease in microvascular density (Figure $5 B)$. During the early stage, within 4 hours, the decrease in $K^{\text {trans }}$ values in the Z-GP-DAVLBH-treated group could be mainly attributable to the reduced perfusion, which was caused by the loss of vessel integrity and hemorrhaging in both the tumor core and peripheral rim (Figure 5B).

The apparent diffusion coefficient (ADC) values reflect the therapy-induced changes in cellularity and water diffusion in proportion to tissue necrosis (25), which were detected simultaneously by DW-MRI to evaluate the antitumor efficacy of
Z-GP-DAVLBH. We found that Z-GP-DAVLBH $(2.0 \mu \mathrm{mol} / \mathrm{kg})$ significantly increased the ADC values to a similar magnitude in the tumor core and the rim (Figure 5A). These effects were consistent with the histological results, where Z-GP-DAVLBH $(2.0 \mu \mathrm{mol} / \mathrm{kg})$ induced dramatic apoptosis of tumor cells surrounding tumor vessels indicated by TUNEL staining at 4 hours through the "bystander" effect and subsequently increased the tumor necrotic area from 2 to 6 days (Figure 5B). Ultimately, the entire tumor underwent necrosis and no cancer cells were detectable from day 12 to day 18 (Figure $5 \mathrm{~B}$ ), leading to the subsequent decrease in ADC values (Figure 5A). In contrast, Z-GP-DAVLBH did not change the $K^{\text {trans }}$ values of normal muscles during the entire therapeutic period (Supplemental Figure 8), indicating that Z-GP-DAVLBH selectively disrupts the tumor vasculature and has no significant effect on normal tissues. Taken together, these results demonstrate that Z-GP-DAVLBH selectively disrupts tumor vessels in both the tumor core and periphery, inducing tumor regression and elimination of the viable rim.

Z-GP-DAVLBH exhibits enhanced antitumor effects and reduced toxicity compared with DAVLBH. We subsequently determined whether targeting tumor pericytes to disrupt tumor vessels by Z-GP-DAVLBH could achieve enhanced antitumor efficacy compared with using DAVLBH, which primarily targets tumor vascular ECs. First, we confirmed that FAP $\alpha$ was overexpressed in the stromal cells of multiple tumor xenografts $(13,26)$ (Supplemental Figure 9A), thus providing the rationale for using these models to evaluate the antitumor efficacy of Z-GP-DAVLBH. The initial experiments evaluating the antitumor effect of Z-GP-DAVLBH were performed on MDA-MB-231 tumor xenografts. We found that DAVLBH inhibited tumor growth by approximately $60 \%$ at $0.5 \mu \mathrm{mol} / \mathrm{kg}$ and induced tumor regression at $1.0 \mu \mathrm{mol} / \mathrm{kg}$. However, 3 of 6 mice did not tolerate the $1.0 \mu \mathrm{mol} / \mathrm{kg}$ treatment and died during treatment, while the remaining 3 mice showed prompt tumor regrowth upon cessation of treatment. In contrast, $0.5 \mu \mathrm{mol} /$ $\mathrm{kg}$ Z-GP-DAVLBH effectively inhibited tumor growth by inducing a partial response (PR) in 5 of 6 mice. Notably, Z-GP-DAVLBH administered at 1.0 or $2.0 \mu \mathrm{mol} / \mathrm{kg}$ greatly reduced tumor size, and all mice showed complete response (CR) after 7 injections. No tumor recurrence was observed for up to 7 weeks. This result is in sharp contrast to that with Boc-AP-DAVLBH, which only inhibited tumor growth by $15 \%$ at $2.0 \mu \mathrm{mol} / \mathrm{kg}$ (Figure $6 \mathrm{~A}$ ). These results suggest that the antitumor efficacy of Z-GP-DAVLBH is much more potent than that of DAVLBH and that FAP $\alpha$ is required to trigger the in vivo antitumor effects of Z-GP-DAVLBH.

Z-GP-DAVLBH $(2.0 \mu \mathrm{mol} / \mathrm{kg})$ also induced regression of other FAP $\alpha$-overexpressing solid tumors (including HepG2, A549, HeLa, and CNE-2 tumor xenografts as well as xenografts derived from primary invasive ductal carcinoma and primary hepatocellular carcinoma patients) and the eradication of viable rims (Figure 6B and Supplemental Figure 9, B and C). Intriguingly, when administered at $2.0 \mu \mathrm{mol} / \mathrm{kg}, \mathrm{Z}-\mathrm{GP}-\mathrm{DAVLBH}$ still induced the regression of MDA-MB-231 tumors even when the treatment started after the tumor size reached approximately $750 \mathrm{~mm}^{3}, 2,500 \mathrm{~mm}^{3}$, or 4,500 $\mathrm{mm}^{3}$ (Figure 6, C and D, and Supplemental Figure 10).

In addition, the toxicity of Z-GP-DAVLBH toward mice bearing MDA-MB-231 xenograft was validated as much lower than that of DAVLBH. Z-GP-DAVLBH given at $1.0 \mu \mathrm{mol} / \mathrm{kg}$ had a 
A
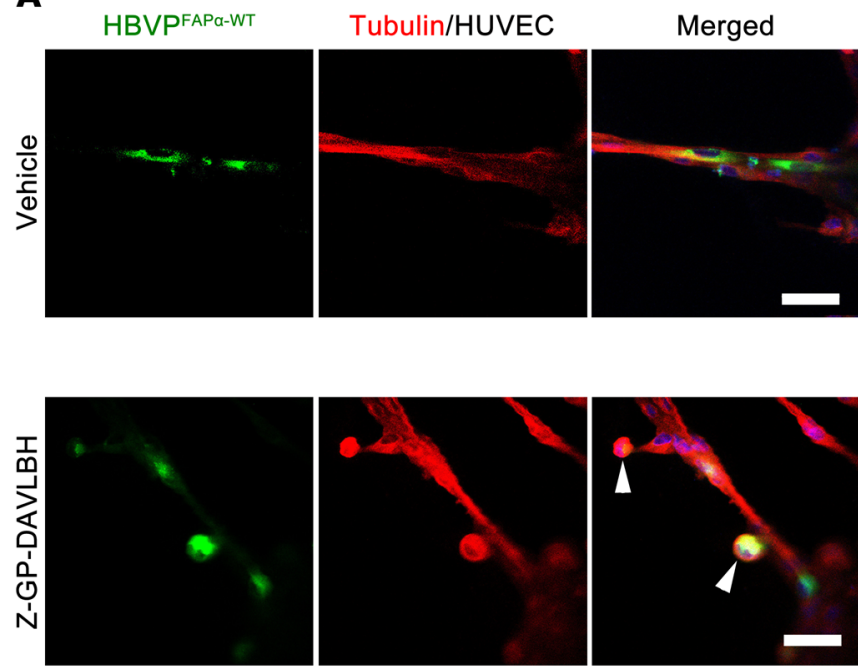

C
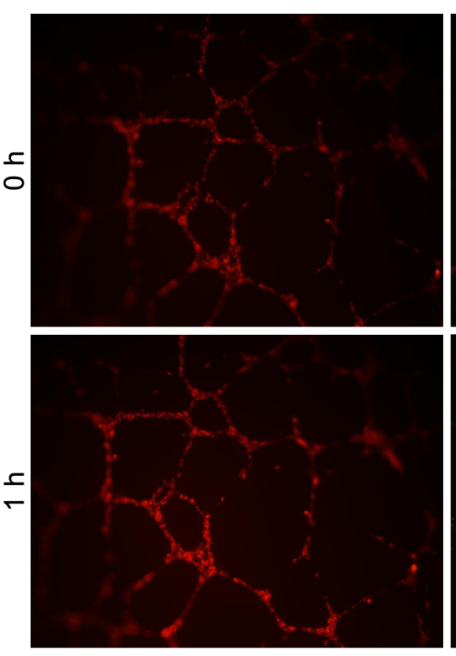

B Z-GP-DAVLBH
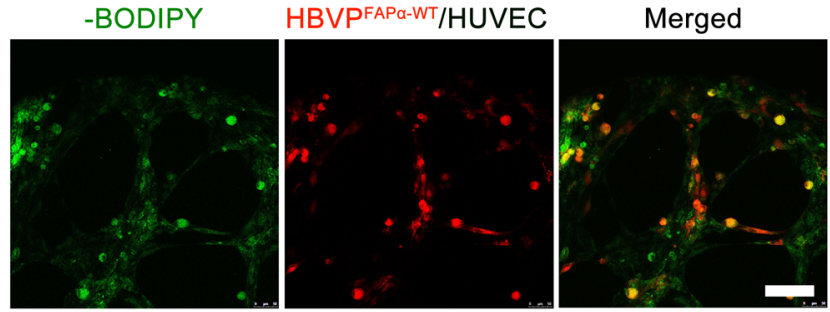

Z-GP-DAVLBH

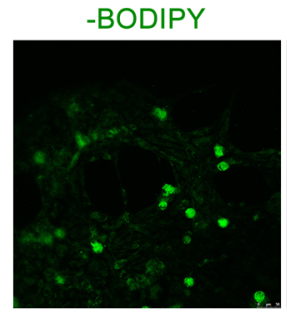

HBVPFAPa-WT/HUVEC
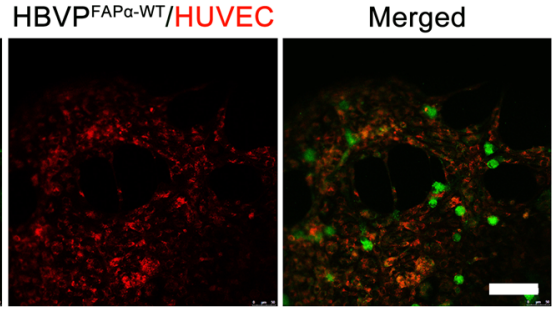

HUVEC/HBVPNC

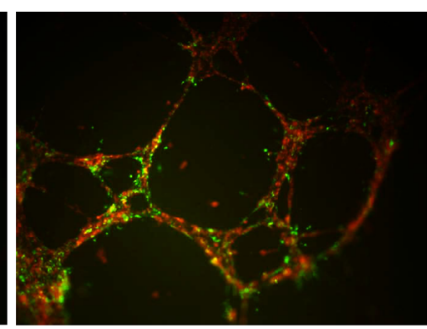

HUVEC/HBVPFAPa-WT
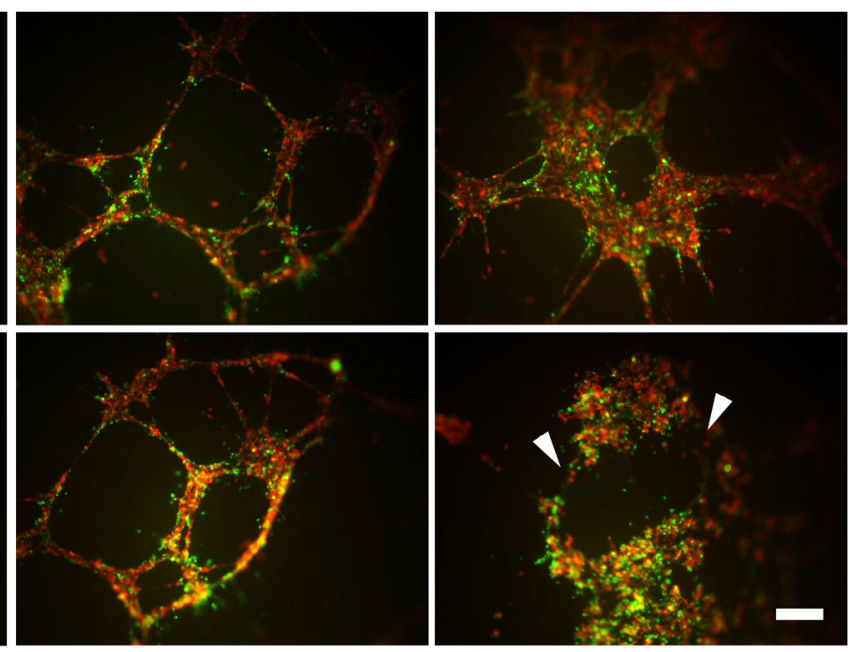

Figure 3. Z-GP-DAVLBH targeting pericytes disrupts pericyte-EC-cocultured tubes. (A) The effect of Z-CP-DAVLBH on the $\beta$-tubulin (red) cytoskeleton of HBVP $^{\text {FAPa-WT }}$ (green, PKH67 staining) covering the HUVEC tubes $(n=3)$. White arrowheads indicate the shrinkage and detachment of HBVPFAP-WT. (B) Evaluation of Z-GP-DAVLBH accumulation in HBVPFAP $\alpha$-WT-HUVEC-cocultured tubes $(n=3$ ). Z-GP-DAVLBH-BODIPY (green, 400 nM) was added to HBVPFAP-WT-HUVECcocultured tubes for 1 hour. HBVPFAPa-WT (red) and HUVECs (red) were stained with PKH 26. (C) The EC- (red) and pericyte-cocultured (green) systems show Z-GP-DAVLBH selectively damages the HBVPFAPa-WT-HUVEC-cocultured tubes (white arrows), but has negligible effect on the HUVEC tubes or HBVPNC-HUVECcocultured tubes $(n=3)$. Scale bars: $100 \mu \mathrm{m}$.

negligible effect on body weight and the number of wbc, which reflect the most common dose-limiting toxicity of vinca alkaloids in the clinic (27-29). However, DAVLBH at the same dosage decreased the number of wbc by $45 \%$ (Supplemental Figure 11, A and B). Similarly, DAVLBH $(1.0 \mu \mathrm{mol} / \mathrm{kg})$ induced significant injuries in bone marrow, while the same dose of Z-GP-DAVLBH showed no significant drug-related toxicity (Supplemental Figure 11C). Moreover, no dramatic pathological changes were observed in the major organs of Z-GP-DAVLBH-treated $(2.0 \mu \mathrm{mol} / \mathrm{kg})$ mice, including heart, liver, spleen, lung, and kidney (Supplemental Figure 11D).

\section{Discussion}

More than 30 years have passed since VDAs were proposed as an attractive approach for cancer therapy. However, tumor resistance to VDAs has hampered their clinical efficacy and drug development. The mechanism underlying tumor resistance to VDAs is not well understood, although several putative mechanisms have been proposed, including hypoxia (30), circulating endothelial progenitor cells $(31,32)$, and tumor-associated macrophages (30). Based on the above mechanisms, various VDA combination therapies have been widely applied, but they either failed to eradicate the tumor viable rim or only partially attenuated treatment resistance, which has reduced the enthusiasm for developing VDAs as anticancer drugs. Here, we demonstrate that specifically targeting pericytes destroys vessels by an FAP $\alpha$-activated prodrug strategy in both the tumor core and the periphery, thus overcoming the VDA treatment resistance, and is of importance for the development of VDAs.

The approach to disrupting tumor vessels primarily focuses on targeting tumor ECs $(1,33)$. The tubulin-binding VDAs selec- 
A
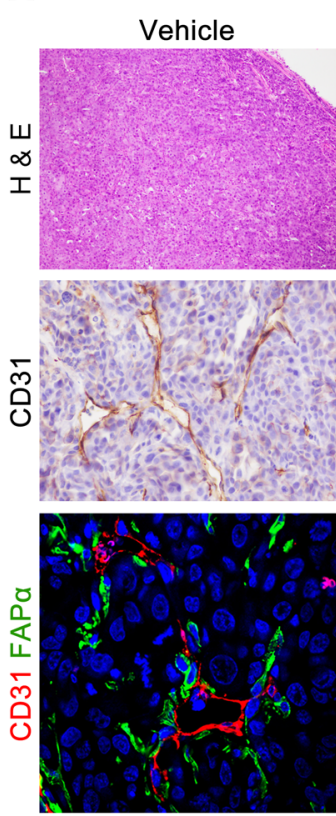

B

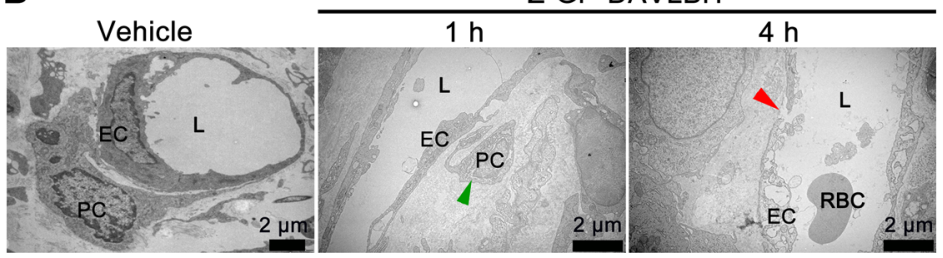

Figure 4. Z-GP-DAVLBH targeting pericytes disrupts vessels in both the tumor core and periphery. Tumors were harvested at 1 hour or 4 hours after MDA-MB-231 xenografts received i.v. injection of $2.0 \mu \mathrm{mol} / \mathrm{kg}$ Z-CP-DAVLBH $(n=5)$. (A) H\&E staining shows vascular disruption in both the tumor core and the periphery; CD31 staining shows "hole-like" disruptions in the vessels (red arrowheads); FAP $\alpha / C D 31$ staining shows the shrinkage and detachment of pericytes (white arrowheads). Tumors were harvested after 4 hours of treatment. Top row scale bars: $200 \mu \mathrm{m}$. Middle row scale bars: $50 \mu \mathrm{m}$. Bottom row scale bars: $50 \mu \mathrm{m}$. (B) The transmission electron microscope images show the effects of Z-GP-DAVLBH on vessels in the tumor periphery. The green arrowhead indicates the shrinkage and detachment of tumor pericyte; the red arrowhead indicates a "gap" disruption between 2 ECs. Scale bars: $2 \mu \mathrm{m}$

tively target tumor ECs by exploiting their dependence on the tubulin cytoskeleton to maintain cell shape $(1,33)$. However, the maturity of vessels, in terms of its investment with pericytes (34), attenuated the efficacy of VDAs. Vessels in the tumor center and periphery have a distinctly different maturity, in which the tumor periphery is supplied by more mature and stable vessels and therefore is not susceptible to treatment with the classical VDA $(7,35)$. Our results also demonstrate that the capillary tubes covered with pericytes and vessels with higher pericyte coverage in the tumor periphery are resistant to DAVLBH, which facilitates the survival of tumor cells in the rim after DAVLBH treatment. These effects may be associated with the fact that pericytes provide survival signals for ECs to protect tumor vessels from chemotherapy (36). Notably, we found that Z-GP-DAVLBH, a pericyte-targeting FAP $\alpha$-activated VDA prodrug, disrupts the pericyte-stabilized capillary tubes and tumor marginal vessels, causing entire tumor hemorrhage and the eradication of the via- ble rim. These findings substantiate the concept that pericytes are an important factor conferring tumor resistance to VDAs and also provide insights into the development of VDAs, including the concept that shifting the target of VDA from tumor vessel ECs to pericytes is an effective strategy for overriding VDA therapy resistance.

Targeting pericytes for anticancer therapy involves 2 main approaches. One way is to construct a drug delivery carrier that targets pericyte-overexpressed markers (NG2 proteoglycan, aminopeptidase A $[\mathrm{APA}]$ ) to enhance the delivery efficacy of conventional anticancer drugs (docetaxel and doxorubicin), such as nanoparticles and liposomes conjugated to pericyte-targeting peptides $(37,38)$. The other way is to target the pericyte itself by blockade of PDGFR- $\beta$ on the pericyte membrane, including aptamers competing against PDGF (39) and PDGFR- $\beta$ inhibitors $(40,41)$. As NG2, APA, and PDGFR- $\beta$ are also overexpressed in CAFs (42-44), the effect of these 2 pericyte-targeting approaches on CAFs is still unclear. Our study complements a simple approach to targeting tumor vessel pericytes by $\mathrm{FAP} \alpha$-activated prodrug strategy, utilizing the property of these cells that highly express FAP $\alpha$. The FAP $\alpha$-activated prodrug strategy is to conjugate an active compound (parent drug) with peptides to form an inactive prodrug, which can later be activated by cells overexpressing FAP $\alpha$. This approach can increase the target probability of a parent drug to FAP $\alpha$-expressing cells, such as tumor vessel pericytes or CAFs. Previous studies revealed that an FAP $\alpha$-activated prodrug strategy is an effective way to target CAFs. Because of the low proliferative index of CAFs, the parent drugs of these prodrugs are cytotoxic thapsigargin and promelittin, which induce cell apoptosis in a proliferation-independent manner $(13,14,16)$. In the present study, we used a proliferation-dependent VDA as the parent drug to establish the FAP $\alpha$-activated prodrug Z-GP-DAVL$\mathrm{BH}$, and this prodrug was demonstrated to selectively target tumor pericytes but not CAFs, indicating that targeting tumor pericytes to disrupt tumor vessels dominates the antitumor effect of Z-GP-DAVLBH. This study extends the understanding of the FAP $\alpha$-activated prodrug, which can achieve either tumor pericyte or fibroblast targeting, depending on the selectivity of the parent drug toward these 2 cells. The selection of a parent drug, which affects the target and the mechanism of action of the prodrug, is one of the most important factors for FAP $\alpha$ activated prodrug strategy.

The therapeutic strategy that targets tumor pericytes is a double-edged sword because of the controversial role of pericytes in tumor metastasis. On one hand, pericytes surrounding tumor vessels play a vital role in promoting tumor vascular normalization, hampering tumor metastasis (45). Depletion of pericytes in tumor vessels by genetic targeting or PDGFR inhibitors facilitated tumor metastasis $(46,47)$. On the other hand, pericytes are required for promoting tumor metastasis by interacting with tumor-associated macrophages (48) or transiting to fibroblasts (49). Consistently, our studies showed that Z-GP-DAVL$\mathrm{BH}$ induced a remarkable reduction of lung metastasis in the MDA-MB-231 breast cancer orthotopic transplantation xenografts. In addition, Z-GP-DAVLBH also caused dramatic necro- 
A Baseline

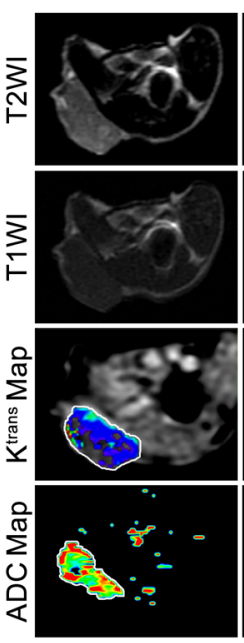

B
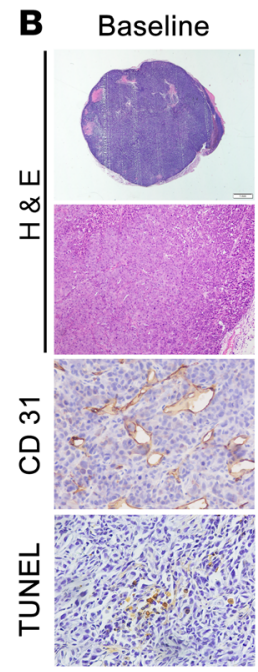

$4 \mathrm{~h}$

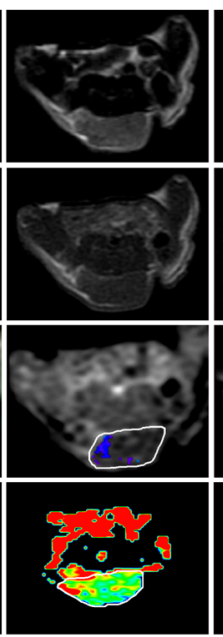

$4 \mathrm{~h}$
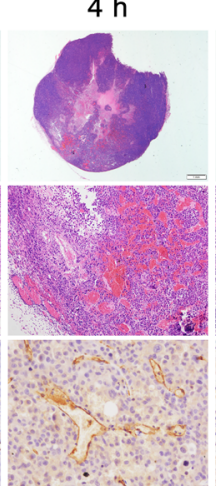

2 days

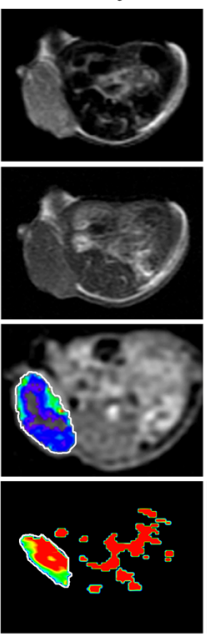

2 days
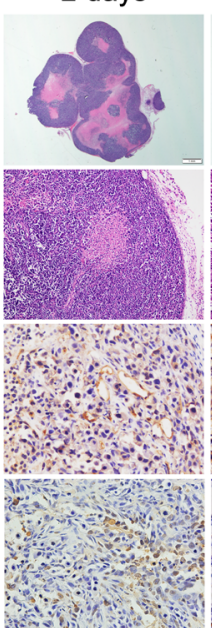

6 days
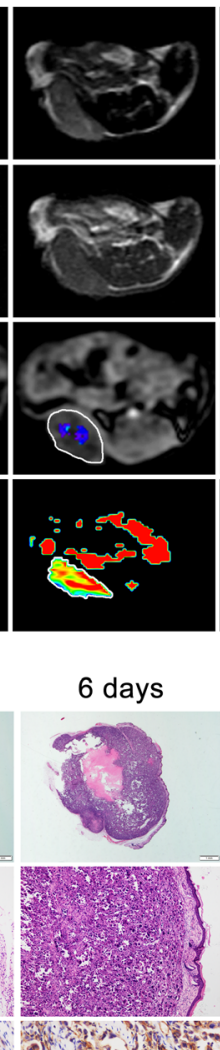

12 days

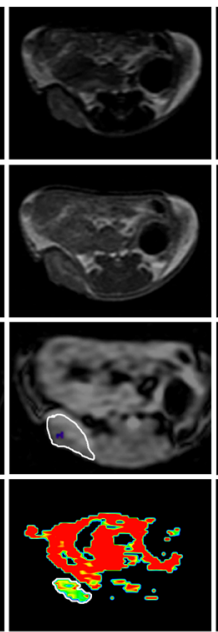

12 days
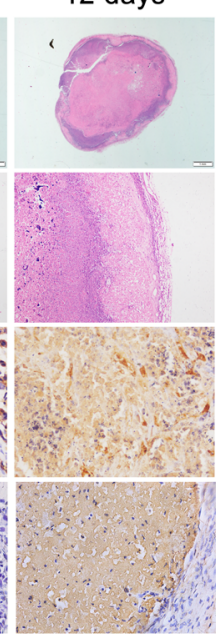

18 days

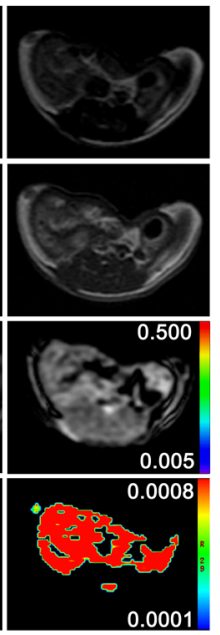

18 days
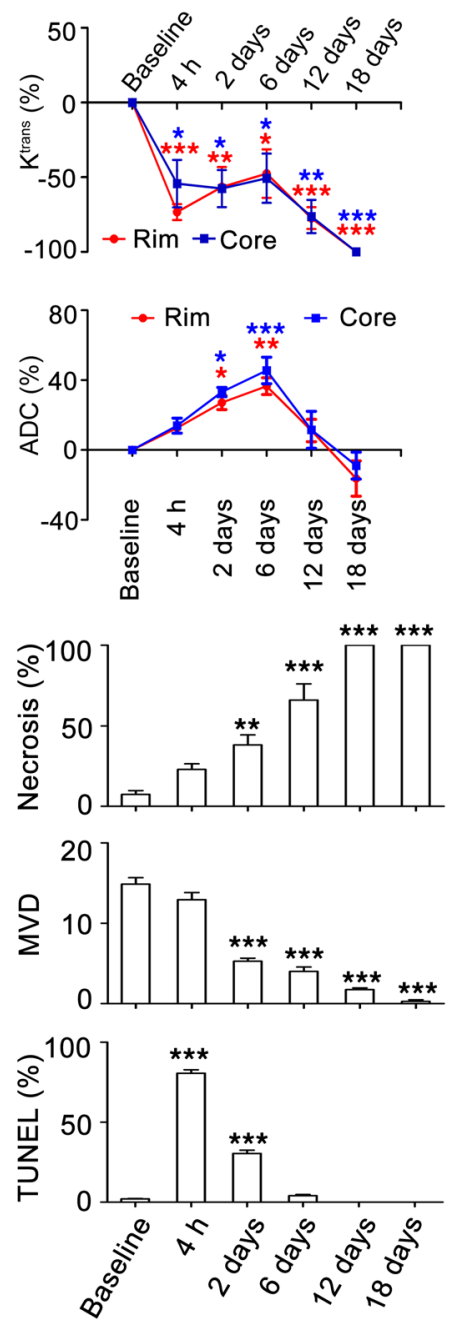

Figure 5. MRI monitoring of the vascular disruption effects of Z-GP-DAVLBH on MDA-MB-231 xenografts. (A) MRI images of tumors throughout therapy with Z-GP-DAVLBH (left). The white rings indicate the tumors. Quantification of $K^{\text {trans }}$ and ADC values is shown (right, $n=5$ ). The mice bearing MDA-MB-231 xenografts received i.v. injection of Z-GP-DAVLBH $(2 \mu \mathrm{mol} / \mathrm{kg})$ once every 2 days. (B) H\&E staining shows increased necrosis in both the tumor core and rim accompanied by prolonged treatment. The entire tumor was necrotic after 12 days of treatment. CD31 staining shows decreased microvascular density (MVD), and tumor sections stained with TUNEL show apoptotic tumor cells. Tumors were harvested at each MRI examination time point. Three images were obtained per tumor, and the quantification of images is shown $(n=5)$. Data are shown as mean $\pm \mathrm{SEM} .{ }^{*} P<0.05 ;{ }^{* *} P<0.01 ;{ }^{* * *} P<0.001$ versus baseline group (1-way ANOVA with Tukey's post hoc comparison).

sis of metastatic lung tumors (Supplemental Figure 12). However, the underlying mechanism of Z-GP-DAVLBH inhibiting tumor metastasis is still unclear and needs further investigation.

Vinca alkaloids are commonly used in cancer therapy (50). However, they lack selectivity for tumor cells versus normal cells and consequently produce severe adverse effects, such as myelosuppression (50). Our results indicate that Z-GP-DAVLBH has more potent antitumor activity and fewer adverse effects than vinblastine sulfate (VLB) or DAVLBH. Because FAP $\alpha$ is overexpressed selectively in pericytes and CAFs in most human epithelial cancers and tumor xenografts, the FAP $\alpha$-activated prodrug may specifically exert pericytetargeting antitumor effects in a variety of malignant carcinomas. Indeed, Z-GP-DAVLBH has a broad spectrum of anticancer activity and induces tumor regression in multiple FAP $\alpha^{+}$tumor xenografts. Z-GP-DAVLBH also demonstrates potent antitumor efficacy in
$\mathrm{FAP}^{+}$primary invasive ductal carcinoma and primary hepatocellular carcinoma patient-derived xenograft models that have been considered suitable for predicting clinical responses to anticancer drugs (51). These results indicate that Z-GP-DAVLBH may achieve great efficacy when evaluated in FAP $\alpha$-overexpressing solid tumors in the clinic.

In summary, this study provides an innovative therapeutic approach to combating VDA treatment resistance through transforming the VDA target from tumor ECs to tumor pericytes by an FAP $\alpha$-activated prodrug strategy. With the unique mechanism of action, Z-GP-DAVLBH can be developed into a promising new VDA for the treatment of FAP $\alpha$-overexpressing cancers.

\section{Methods}

Reagents. VLB (catalog N2255) and ValboroPro (catalog B3941) were obtained from ApexBio. The rabbit anti-FAP $\alpha$ primary antibody was 
a gift from Hyung-Ok Lee, Hossein Borghaei, and John Cheng (Fox Chase Cancer Center, Philadelphia, Pennsylvania, USA; 1:1,000 dilution for immunofluorescence staining and 1:3,000 dilution for Western blotting) (13). The human FAP $\alpha$ cDNA clone (catalog SC117372) and pCMV6-entry vector (catalog PS100001) were obtained from Origene Technologies. Lipofectamine LTX Reagent with PLUS Reagent (catalog 15338-100) was purchased from Invitrogen. rhFAP $\alpha$ (catalog 3715-SE), rhDPP-IV (1180-SE), rhPEP (4308-SE), and goat anti-CD31 primary antibody (catalog AF3628, 1:200 dilution for immunofluorescence and immunohistochemical staining) were obtained from R\&D Systems. The DeadEnd Colorimetric TUNEL System (catalog G7130) was obtained from Promega Corp. Mouse anti-Ki67 (catalog 9449, 1:200 dilution for immunohistochemical staining), rabbit anti- $\beta$-tubulin (catalog 2128, 1:200 dilution for immunofluorescence staining), mouse anti- $\beta$-actin primary antibody (catalog 3700, 1:1000 dilution for Western blotting), and HRPconjugated anti-rabbit (catalog 7076, 1:2,000 dilution for Western blotting) and anti-mouse (catalog 7074, 1:2,000 dilution for Western blotting) antibodies were purchased from Cell Signaling Technology. Alexa Fluor 594-conjugated donkey anti-goat IgG (H+L) (catalog A-11058, 1:1,000 dilution for immunofluorescence staining), Alexa Fluor 488-conjugated donkey anti-rabbit $\operatorname{IgG}(\mathrm{H}+\mathrm{L})$ (catalog A-21206, 1:1,000 dilution for immunofluorescence staining) and Alexa Fluor 594-conjugated donkey anti-rabbit IgG $(\mathrm{H}+\mathrm{L})$ (catalog A-21207, 1:1,000 dilution for immunofluorescence staining) were purchased from Life Technologies (Invitrogen). Rhodamine phalloidin (catalog R415) and enhanced chemiluminescence reagent (cata$\log 32132$ ) were purchased from Thermo Fisher Scientific. The Tubulin Polymerization Assay Kit (catalog BK011P) was obtained from Cytoskeleton. The 17 $\beta$-estradiol slow release pellet (catalog SE-121, 60 -day release, $0.36 \mathrm{mg} / \mathrm{pellet}$ ) was obtained from Innovative Research of America. Matrigel (catalog 354248) was purchased from Corning. DAPI (catalog D9542), PKH 26 (catalog SLBL9605V), PKH 67 (catalog SLBNO256V), pentobarbital sodium salt (catalog P3761), and other agents were all purchased from Sigma-Aldrich.

Synthesis and characterization of test compounds. The detailed synthetic processes of DAVLBH, Z-GP-DAVLBH, Boc-AP-DAVLBH, and Z-GP-DAVLBH-BODIPY are described in Supplemental Methods and Supplemental Figure 13. Liquid chromatography-mass spectrometry (LC-MS) data, ${ }^{1} \mathrm{H}$-NMR signals, and ${ }^{13} \mathrm{C}$-NMR signals are in agreement with the proposed structures for DAVLBH, Z-GP-DAVL$\mathrm{BH}$, Boc-AP-DAVLBH, and Z-GP-DAVLBH-BODIPY. The purity of these compounds was validated as more than $95 \%$, and they were dissolved in DMSO to obtain a 20-mM stock solution.

Cells and cell culture. The human embryonic kidney cell line HEK 293T, the mouse embryo fibroblast cell line BALB/3T3, the human breast adenocarcinoma cell line MDA-MB-231, the human hepatocellular carcinoma cell line HepG2, the human cervix adenocarcinoma cell line HeLa, and the human lung carcinoma cell line A549 were obtained from ATCC, and the human nasopharyngeal carcinoma cell line CNE-2 was a gift from Jian Hong (Southern Medical University). The above cell lines were cultured in DMEM supplemented with 10\% FBS (Life Technologies). HUVECs and HBVPs were obtained from ScienCell Research Laboratories and cultured in EC medium (ECM) and pericyte medium (PM), respectively. Excluding CNE-2, the other cell lines used in this study were authenticated as having no crosscontamination of other human cell lines using the STR Multi-Amplifica- tion Kit (Microreader 21 ID System). All cell lines tested negative for mycoplasma using the Mycoplasma Detection Set (M\&C Gene Technology).

Generation of FAP $\alpha$-transfected cells. The 5 site-directed mutagenesis sequences of FAP $\alpha$ were synthesized by Beijing Genomics Institute (Beijing, China) and were cloned into HindIII, HindIII/ClaI, HindIII/ ClaI, MluI or MluI /SalI-digested full-length WT human FAP $\alpha$ plasmids, respectively. The FAP $\alpha$ mutations were verified by DNA sequencing. The WT FAP $\alpha$ plasmid was transfected into HEK-293T, HBVPs, and BALB/ 3T3 cells using Lipofectamine LTX with PLUS Reagent to generate HEK-293T ${ }^{\text {FAPa-WT }}$, HBVP ${ }^{\text {FAP } \alpha-W T}$, and BALB/3T3 ${ }^{\text {FAPa-WT }}$ cells. The 5 mutant FAP $\alpha$ plasmids were transfected into HEK-293T cells to generate HEK293T ${ }^{\mathrm{R} 123 \mathrm{~A}}$, HEK-293T ${ }^{\mathrm{E} 203 \mathrm{~A}}$, HEK-293T ${ }^{\mathrm{E} 204 \mathrm{~A}}$, HEK-293T ${ }^{\mathrm{Y} 656 \mathrm{~F}}$, and HEK$293 T^{\mathrm{N} 704 \mathrm{~A}}$ cells. Cells were transfected with the pCMV6-entry vector to generate $\mathrm{HEK}-293 \mathrm{~T}^{\mathrm{NC}}, \mathrm{HBVP}^{\mathrm{NC}}$, and $\mathrm{BALB} / 3 \mathrm{~T}^{\mathrm{NC}}$ cells as the negative controls. After transfection, the cells were cultured at $37^{\circ} \mathrm{C}$ for another 48 hours, and transfection efficiency was determined by Western blotting. See complete unedited blots in the supplemental material.

FAP $\alpha, D P P-I V$, and PEP enzymatic assays. The calibration curve of DAVLBH was first constructed by the addition of VLB as an internal standard $(1 \mu \mathrm{M})$ to $50 \mathrm{mM}$ Tris buffer. $\mathrm{rhFAP} \alpha(5 \mathrm{ng} / \mathrm{ml})$ was incubated with various concentrations of Z-GP-DAVLBH $(0.625,1.25,2.5,5.0$, 10.0, 20.0 and $40.0 \mu \mathrm{M})$ at $37^{\circ} \mathrm{C}$ for 1 hour. To compare the hydrolysis effects, $5 \mathrm{ng} / \mathrm{ml}$ of $\mathrm{rhFAP} \alpha$, rhDPP-IV, and rhPEP was incubated with $10 \mu \mathrm{M} Z$ Z-GP-DAVLBH or Boc-AP-DAVLBH at $37^{\circ} \mathrm{C}$ for 1 hour. The supernatants were deproteinated with 3 -fold (v/v) acetonitrile/0.1\% formic acid and centrifuged at 3,000 $g$ for 15 minutes. The resulting supernatants were analyzed for drug concentration by LC coupled to a VEVO-QTOF MS (Waters) equipped with a BEH-C18 reverse-phase column $(1.7 \times 250 \mathrm{~mm}, 1.7 \mu \mathrm{m}$, catalog 0265352581, Waters $)$ and operated in ESI-positive ion mode. Each compound was identified by the individual extracted ion chromatogram, and the areas of DAVLBH were converted into a ratio with the internal standard. The concentration data were analyzed using the calibration standards and using the enzymatic reaction system. The kinetic constants $K_{m}, V_{\max }$, and $K_{c a t}$ were calculated according to Michaelis-Menten with a nonlinear regression model using GraphPad Prism 5.0 software.

Analysis of FAP $\alpha$ enzymatic activity in cell lines and drug concentration in tumor tissues. Calibration standards of DAVLBH, coupled with an internal standard, were performed before analysis. The cell lines were seeded in 6-well plates and cultured for 24 hours; the culture medium was then replaced with PBS containing Z-GP-DAVLBH (10 $\mu \mathrm{M})$. After a 2-hour incubation period, the above supernatants were collected. For the inhibitory study, $100 \mu \mathrm{M}$ ValboroPro was added to the reactive system and preincubated for 1 hour. All of the above collected supernatants were then deproteinated with 3-fold (v/v) acetonitrile/0.1\% formic acid and centrifuged at 3,000 $g$ for 15 minutes. To detect the drug concentration in tumor, Z-GP-DAVLBH was i.v. injected into mice bearing MDA-MB-231 tumors with a tumor size of approximately $500 \mathrm{~mm}^{3}$ at a dose of $2 \mu \mathrm{mol} / \mathrm{kg}$. At the expected time after injection, 5 mice were anesthetized and killed. The tumors were obtained, weighed, and then homogenized with PBS $(1: 4, w / v)$ at $4^{\circ} \mathrm{C}$. The tumor homogenate was treated in a manner similar to that of the cell culture supernatants as described above. The above samples were analyzed for drug concentration by LC-MS.

Tubulin polymerization assay. The tubulin polymerization assay was performed using a Tubulin Polymerization Assay Kit according to the manufacturer's instructions (Cytoskeleton). Tubulin polymeriza- 

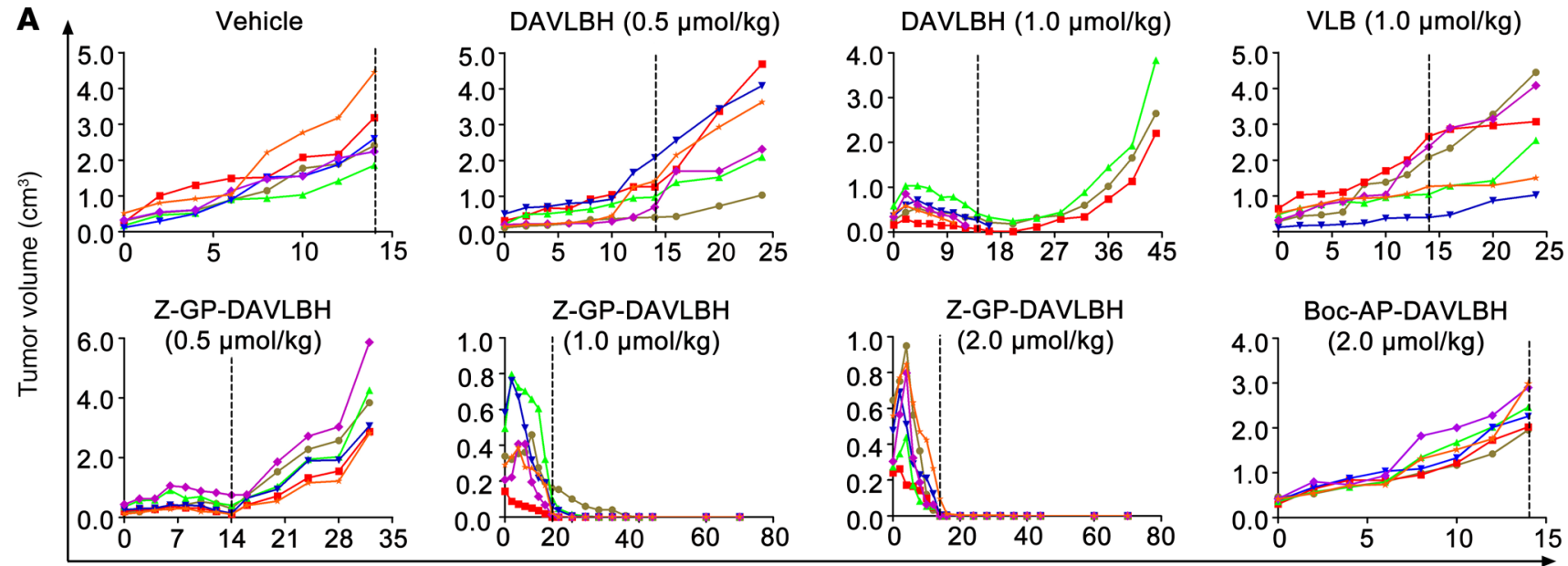

Days after treatment

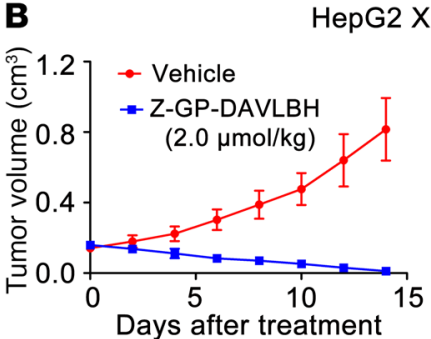

HeLa Xenograft

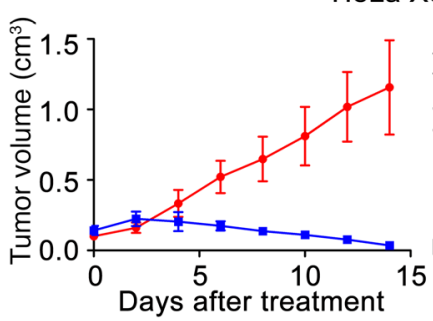

Invasive ductal carcinoma PDX

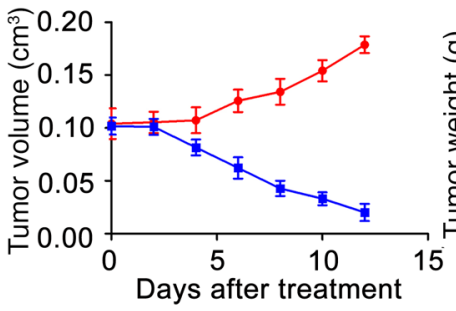

C
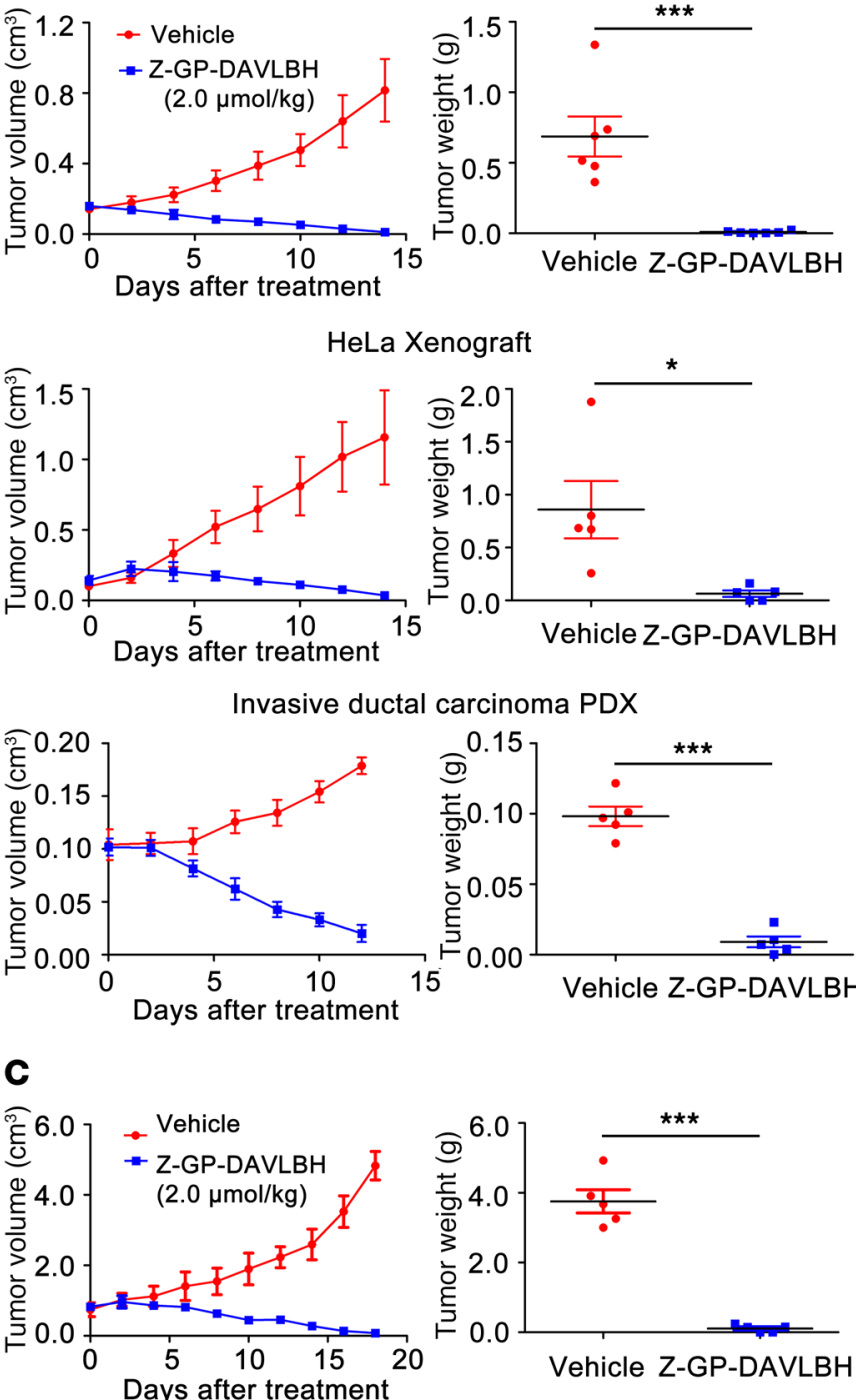

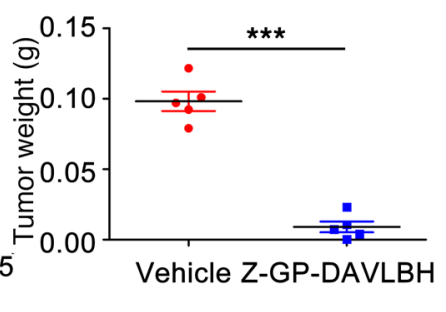

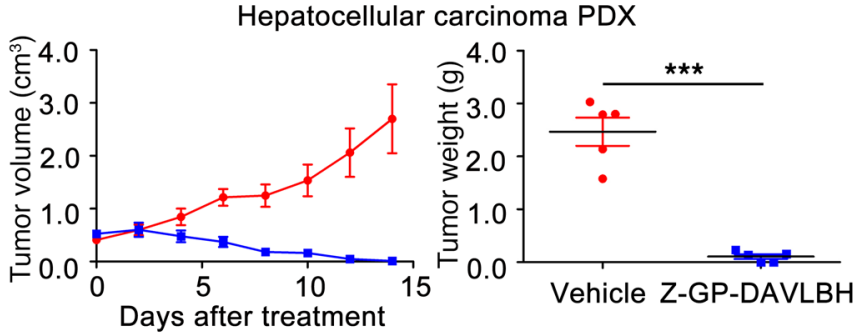

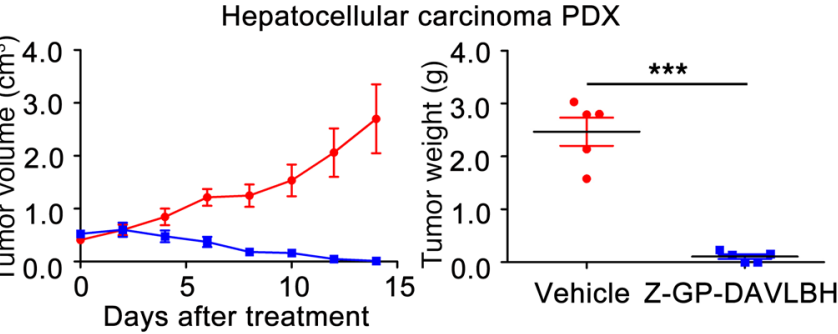

D
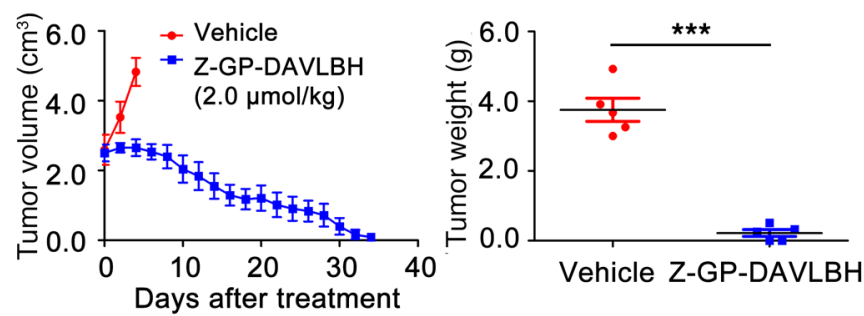

CNE-2 Xenograft

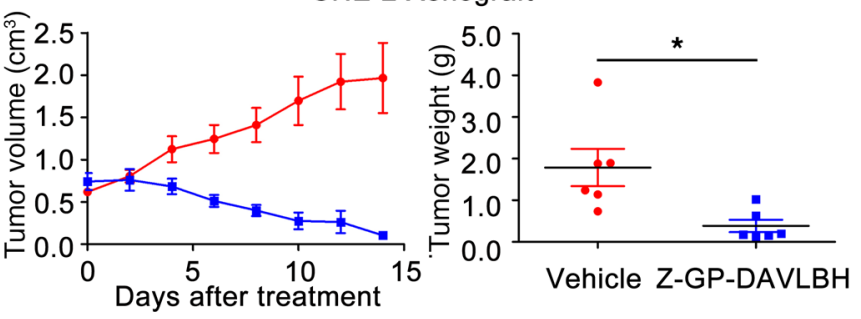

Hepatocellular carcinoma PDX

Vehicle Z-GP-DAVLBH 
Figure 6. Antitumor effect of Z-GP-DAVLBH in multiple tumor xenografts. (A) Comparative therapeutic efficacy of VLB, DAVLBH, Z-GP-DAVLBH, and Boc-AP-DAVLBH in MDA-MB-231 xenografts. Mice bearing MDA-MB-231 xenografts received an i.v. injection of saline (containing 1\% DMSO), VLB, DAVLBH, Z-GP-DAVLBH, or Boc-AP-DAVLBH once every other day for 14 days. Each curve represents the growth of a single tumor in an individual mouse. The dotted vertical lines denote the final day of dosing. (B) Detection of the anticancer spectrum of Z-CP-DAVLBH. Mice bearing HepG2 xenografts ( $n=5$ ), A549 xenografts $(n=5)$, HeLa xenografts $(n=5)$, CNE-2 xenografts $(n=6)$, invasive ductal carcinoma patient-derived xenografts (PDX, $n=5)$, and hepatocellular carcinoma PDX $(n=5)$ received an i.v. injection of Z-GP-DAVLBH $(2.0 \mu \mathrm{mol} / \mathrm{kg})$ once every other day for 12 or 14 days. Mice in the vehicle group received saline (containing 1\% DMSO) only. (C and $\mathbf{D}$ ) The antitumor effects of Z-GP-DAVLBH in the large MDA-MB-231 tumor experiment. Mice bearing MDAMB-231 xenografts received $2.0 \mu \mathrm{mol} / \mathrm{kg}$ i.v. injection of Z-GP-DAVLBH or saline containing $1 \%$ DMSO once every other day for 16 or 32 days, until the tumor volume reached approximately (C) $750 \mathrm{~mm}^{3}(n=5)$ or $(\mathbf{D}) 2,500 \mathrm{~mm}^{3}(n=5)$. For $\mathbf{B}-\mathbf{D}$, the tumor volume (left) and the tumor weight (right) are shown. The results are expressed as mean $\pm \mathrm{SEM}$. ${ }^{*} P<0.05 ;{ }^{* *} P<0.01 ;{ }^{* *} P<0.001$ versus the vehicle group based on a 2 -tailed unpaired $t$ test.

tion was indicated by the fluorescence value and was monitored with the following kinetic program: excitation at $360 \mathrm{~nm}$ and emission at $420 \mathrm{~nm}$, with 61 cycles of 1 reading per minute for 90 readings.

$3 D$ cocultures. The cells were labeled with PKH 26 for red fluorescence and PKH 67 for green fluorescence. Next, $2 \times 10^{4}$ labeled ECs were mixed with or without $4 \times 10^{3}$ labeled pericytes suspended in serum-free medium (ECM/PM, 5:1) and seeded in Matrigelcoated 96-well plates. The cells were incubated for 6 hours to allow for $3 \mathrm{D}$ cocultured tube formation. For the tube-disruption assay, the supernatants of the cocultured tubes were replaced with fresh serumfree medium containing $500 \mathrm{nM}$ DAVLBH, Boc-AP-DAVLBH, or Z-GP-DAVLBH. Images were obtained with an Olympus IX70 inverted fluorescence microscope 1 hour after treatment. For the drug accumulation assays, the supernatants of the cocultured tubes were replaced with fresh serum-free medium containing $400 \mathrm{nM}$ Z-GP-DAVLBHBODIPY or free BODIPY for 1 hour, followed by washing with serumfree medium for 10 minutes. Cells were observed under a Zeiss LSM 700 confocal microscope. The assay was performed in triplicate.

Establishment of tumor xenografts. Four- to six-week-old NU/ NU nude mice and NOD/SCID mice were obtained from Vital River Laboratory Animal Technology Co. The primary invasive ductal carcinoma and primary hepatocellular carcinoma surgical specimens were obtained intraoperatively from the Cancer Center of Guangzhou Medical University. The information for primary invasive ductal carcinoma and primary hepatocellular carcinoma patients is in Supplemental Table 1. After obtaining surgical specimens, fresh tumor tissues were kept on ice in RPMI 1640 and then minced into 3- to 5- $\mathrm{mm}^{3}$ fragments. The invasive ductal carcinoma tissues were orthotopically transplanted into the fourth mammary fat pads of female NOD/SCID mice to establish the invasive ductal carcinoma patient-derived xenografts. Before transplantation, the mice were anesthetized and s.c. implanted with $17 \beta$-estradiol supplementation (52). The hepatocellular carcinoma tissues were subcutaneously inoculated on the backs of male NOD/SCID mice to establish the hepatocellular carcinoma PDX. Two xenografts were established after 4 weeks of grafting and then subsequently passed from mouse to mouse to expand the number of xenografts. To establish the tumor xenograft models of MDA-MB-231, HepG2, A549, HeLa, and CNE- 2 cell lines, $2 \times 10^{6}$ cells of each cell line in $200 \mu \mathrm{l}$ of a $50 \%$ mixture of Matrigel were inoculated s.c. into the backs of female (HeLa and MDA-MB-231 cells) or male (other cell lines) NU/NU nude mice. MDA-MB-231 cells $\left(1 \times 10^{6} /\right.$ mouse $)$ were inoculated orthotopically into the fourth mammary fat pads of female NU/NU mice to establish the orthotopic transplantation tumor.

In vivo assay. When the tumor xenograft size reached between 200 and $500 \mathrm{~mm}^{3}$, tumor-bearing mice were randomized to the appropriate groups ( 5 to 6 mice per group) by the Research Randomizer (http:// www.randomizer.org). The mice were i.v. injected with test compounds once every other day. Mice in the vehicle group received saline (containing 1\% DMSO) only. In the large MDA-MB-231 tumor experiment, mice were dosed in 750-, 2,500- or 4,500- $\mathrm{mm}^{3}$ groups. Mice were then examined for tumor growth and body weight changes. Tumors were measured using a slide caliper (Mitutoyo), and their volumes were calculated using the following formula: $a^{2} \times b \times 0.5$, where $a$ refers to the smaller diameter and $b$ is the diameter perpendicular to $a$. A PR was defined as a volume regression of more than $50 \%$, but with a measurable tumor size $\left(>10 \mathrm{~mm}^{3}\right)$ present at all times. A CR was defined as follows: tumor could not be detected, tumor volumetric measurements could not be performed $\left(<10 \mathrm{~mm}^{3}\right)$, and histologic examination of the tumor tissue did not show evidence of a tumor (53). At the end of the experiments, the mice were anesthetized by i.p. injection of $5 \mathrm{ml} / \mathrm{kg}$ of $1 \%$ pentobarbital sodium salt, and blood was collected by intracardiac puncture. Blood samples were anticoagulated with EDTA-2K, and wbc were immediately counted using a Unicel DxH 800 automated hematology analyzer (Beckman Coulter). Tumors were removed, weighed, and photographed. The major organs, such as the heart, liver, spleen, lung, and kidney, as well as the femora, were removed and fixed in $4 \%$ paraformaldehyde until pathological examination.

Histology and immunohistochemical analysis. Fixed tumor tissues and mouse organs were embedded in paraffin, sectioned at a thickness of 5 $\mu \mathrm{m}$, and stained using a standard H\&E procedure. Immunohistochemistry was performed on deparaffinized tumor sections, and antigen retrieval was performed in $10 \mathrm{mM}$ sodium citrate buffer ( $\mathrm{pH}$ 6.0). Slides were then blocked with 5\% BSA and incubated with anti-CD31 or anti-Ki67 overnight at $4^{\circ} \mathrm{C}$. Slides were incubated with HRP-conjugated secondary antibodies and stained using a DAB kit, followed by counterstaining with hematoxylin. TUNEL staining was performed using the DeadEnd Colorimetric TUNEL System Kit according to the manufacturer's instructions (Promega Corp.). Slides were visualized using an Olympus BX 53 microscope. Three fields were counted from each slide, and all image analyses were performed using Image Pro Plus 6.0 software.

Immunofluorescence staining for tumor tissues and cell lines. Excised tumors were fixed in $4 \%$ paraformaldehyde overnight and then soaked in a $30 \%$ sucrose solution for 3 days until they were embedded in OCT (Sakura, catalog 4583). Subsequently, 5- $\mu \mathrm{m}$ cryosections were incubated with anti-FAP $\alpha$ and anti-CD31 overnight at $4^{\circ} \mathrm{C}$. The cells were seeded on dishes, and cocultured tubes were fixed in $4 \%$ paraformaldehyde. Anti- $\beta$-tubulin was used as the primary antibody, and rhodamine phalloidin was used to identify F-actin. Subsequently, the slides or dishes were incubated with Alexa Fluor dye-conjugated secondary antibodies and DAPI for cell nuclear staining. The slides or dishes were observed under a Zeiss LSM 700 confocal microscope.

Electron microscopy analysis. Tumor tissues were cut into approximately $30-\mathrm{mm}^{3}$ fragments and fixed with $4 \%$ paraformaldehyde containing $2.5 \%$ glutaraldehyde for 1 hour at $4^{\circ} \mathrm{C}$. The tumor fragments 
were embedded in EPON resin, processed for preparation of ultrathin (70-90 nm) sections, and subsequently stained with uranyl acetate and lead citrate. The grids were examined under a Philips Tecnai 12 or HITACHI H-750O electron microscope.

MRI. All MRI examinations were performed using a 1.5-T MR system (GE Healthcare Signa HDxt) equipped with an 8-channel wrist coil, and the mice were in the supine position. Prior to MRI scanning, the mice were anesthetized, and a 24-gauge catheter was inserted into their tail veins attached to a syringe to allow injection of the contrast compound Gd-DTPA (Magnevist, catalog 1311935, Bayer Schering Pharma). The mice were scanned before and after i.v. injection of Z-GP-DAVLBH $(2.0 \mu \mathrm{mol} / \mathrm{kg})$ at various times. The fast spin echo (FSE) sequence was used to acquire transverse T1-weighted images with the following parameters: repetition/echo time (TR/TE), 400/17.6 $\mathrm{ms}$; matrix size, $256 \times 192$; field of view (FOV), $70 \mathrm{~mm} \times 49 \mathrm{~mm}$; slice thickness, $2 \mathrm{~mm}$; slice gap, $0.2 \mathrm{~mm}$; number of slices, 8 ; number of excitations (NEX), 2. The transverse T2-weighted images had the following parameters: TR/TE, 2040/77.6 ms; matrix size, $256 \times 192$; FOV, $70 \mathrm{~mm} \times 56 \mathrm{~mm}$; slice thickness, $2 \mathrm{~mm}$; slice gap, $0.2 \mathrm{~mm}$; number of slices, 8; NEX, 2. DW-MRI was acquired using a single-shot echoplanar imaging (SSEPI) pulse sequence with thirteen $b$ values $(0,25$, $50,75,100,150,200,400,600,800,1000,1200$, and $\left.1500 \mathrm{~s} / \mathrm{mm}^{2}\right)$; TR/TE, 4000/95 ms; FOV, $9.0 \mathrm{~cm} \times 4.5 \mathrm{~cm}$; matrix size, $96 \times 128$; slice thickness, $2 \mathrm{~mm}$; number of slices, 8 ; slice gap, $0.2 \mathrm{~mm}$; and NEX, 1, 2, $2,3,3,3,4,4,4,6,6,8,10$ corresponding with each $b$ value. The acquisition time for DWI was 3 minutes 48 seconds. DCE-MRI was performed using a 3D fast spoiled gradient-recalled echo (3D-FSPGR) sequence with the following parameters: TR/TE, 40/2.4 ms; matrix size, $128 \times$ 96; FOV, $70 \mathrm{~mm} \times 56 \mathrm{~mm}$; slice thickness, $2 \mathrm{~mm}$; number of slices, 6; slice gap, $0.2 \mathrm{~mm}$; flip angle, $35^{\circ}$; NEX, 1 . Each mouse was catheterized and injected with Gd-DTPA $(0.1 \mathrm{mmol} / \mathrm{kg})$ via tail vein catheter, followed by $0.3 \mathrm{ml}$ of a saline flush at the start of the imaging sequence. The DCE-MRI was continuously scanned for 35 phases with a temporal resolution of 3 seconds. Before DCE-MRI, the baseline T1 was quantitatively mapped using variable flip angles of $3^{\circ}, 6^{\circ}, 9^{\circ}, 12^{\circ}$, and $15^{\circ}$. At each imaging time point, 5 mice that received the same therapeutic regimen were euthanized, and the tumors were excised and processed for histological examination.

MRI analysis. MRI data processing was performed offline at an independent workstation (Advantage Workstation 4.5, GE Healthcare) by a radiologist with 6 years of clinical experience. The region of interest (ROI) was selected on the dorsal normal muscle or on the maximum tumor-containing slice (excluding skin). The DWI data were analyzed using the Functool MADC program, and a biexponential model was used to calculate the ADC (54). The DCE-MRI data were analyzed using the Cinetools program, and the volume transfer coefficient $\left(K^{\text {trans }}\right)$ was calculated based on the Tofts model (55).

Statistics. GraphPad Prism 5.0 software was used for statistical analyses (GraphPad Software Inc.). All data are presented as the mean values with SEM. Significant differences between 2 groups were evaluated using the 2-tailed unpaired $t$ test, and significant differences between more than 2 groups were evaluated using 1-way ANOVA followed by Tukey's post hoc test (as indicated in the figure legends). $P$ values of less than 0.05 were considered significant.

Study approval. The clinical specimens used in this study were approved by the Cancer Center of Guangzhou Medical University Institutional Review Board, and written informed consent was received from participants prior to inclusion in the study. All animal studies were conducted with the approval of the Laboratory Animal Ethics Committee of Jinan University.

\section{Author contributions}

WY and DZ designed and supervised the experiments, analyzed the data, and wrote and revised the manuscript. QY provided critical reading and revision of the manuscript. $\mathrm{MC}$ and $\mathrm{X}$ Lei wrote the manuscript and analyzed the data. HC, ZL, NX, NM, XZ, and $\mathrm{CF}$ generated the chemical compounds. HL provided the clinical samples. MC, X Lei, MH, X Li, QN, JH, and WM performed the animal experiments. CS and LL performed the MRI and analyzed the data. BD provided pathologist review and assessment of clinical and preclinical samples. BW, $\mathrm{WH}, \mathrm{AH}, \mathrm{MC}$, and $\mathrm{AM}$ performed LC-MS analysis. MC, X Lei, QN, MH, and MX performed the $\mathrm{H} \& \mathrm{E}$, immunohistochemical analysis, immunofluorescence staining, electron microscopic analysis, 3D cocultures, and tubulin polymerization assay and their image acquisition. X Lei, $\mathrm{MH}$, and X Li performed cell line studies and Western blotting.

\section{Acknowledgments}

This study was supported by the National Natural Science Foundation of China (U1401225, 81630095, and 81573455), the Natural Science Foundation of Guangdong Province (S2013050014183 and 2013CXZDA006), the Program for New Century Excellent Talents in University, the Pearl River Scholar Funded Scheme (to DZ), and the National Science and Technology Major Project (2017ZX09101003008-008). We gratefully thank Hyung-Ok Lee, Hossein Borghaei, and John Cheng (Fox Chase Cancer Center) for providing the FAPa primary antibody. We thank Jian Hong (Southern Medical University) for providing the CNE-2 cell line. We thank Youwei Zhang (Case Western Reserve University, Cleveland, Ohio, USA), Donghua Yang (St. John's University, New York, New York, USA), and Charles R. Ashby Jr. (St. John's University) for revising the manuscript.

Address correspondence to: Wencai Ye, Dongmei Zhang, or Heru Chen, College of Pharmacy, Jinan University, 601 West Huangpu Road, Guangzhou 510632, China. Phone: 86.20.85220004; Email: chywc@aliyun.com (W. Ye); Phone: 86.20.85222653; Email: dmzhang701@foxmail.com (D. Zhang); Phone: 86.20.38375299; Email: thrchen@jnu.edu.cn (H. Chen).
1. Tozer GM, Kanthou C, Baguley BC. Disrupting tumour blood vessels. Nat Rev Cancer. 2005;5(6):423-435.

2. Blakey DC, et al. Antitumor activity of the novel vascular targeting agent ZD6126 in a panel of tumor models. Clin Cancer Res. 2002;8(6):1974-1983.
3. Liang W, Ni Y, Chen F. Tumor resistance to vascular disrupting agents: mechanisms, imaging, and solutions. Oncotarget. 2016;7(13):15444-15459.

4. Vincent L, et al. Combretastatin A4 phosphate induces rapid regression of tumor neovessels and growth through interference with vascular endothelial-cadherin signaling. J Clin Invest.
2005;115(11):2992-3006.

5. Jockovich ME, et al. Retinoblastoma tumor vessel maturation impacts efficacy of vessel targeting in the LH(BETA)T(AG) mouse model. Invest Ophthalmol Vis Sci. 2007;48(6):2476-2482.

6. Tozer GM, et al. Blood vessel maturation and response to vascular-disrupting therapy in 
single vascular endothelial growth factor-A isoform-producing tumors. Cancer Res. 2008;68(7):2301-2311.

7. Nguyen L, et al. Spatial morphological and molecular differences within solid tumors may contribute to the failure of vascular disruptive agent treatments. BMC Cancer. 2012;12:522.

8. Armulik A, Genové G, Betsholtz C. Pericytes: developmental, physiological, and pathological perspectives, problems, and promises. Dev Cell. 2011;21(2):193-215.

9. Levéen P, Pekny M, Gebre-Medhin S, Swolin B, Larsson E, Betsholtz C. Mice deficient for PDGF B show renal, cardiovascular, and hematological abnormalities. Genes Dev. 1994;8(16):1875-1887.

10. Soriano P. Abnormal kidney development and hematological disorders in PDGF beta-receptor mutant mice. Genes Dev. 1994;8(16):1888-1896.

11. Yu DM, et al. The dipeptidyl peptidase IV family in cancer and cell biology. FEBS J . 2010;277(5):1126-1144.

12. Piñeiro-Sánchez ML, et al. Identification of the 170-kDa melanoma membrane-bound gelatinase (seprase) as a serine integral membrane protease. J Biol Chem. 1997;272(12):7595-7601.

13. Brennen WN, Rosen DM, Wang H, Isaacs JT, Denmeade SR. Targeting carcinoma-associated fibroblasts within the tumor stroma with a fibroblast activation protein-activated prodrug. J Natl Cancer Inst. 2012;104(17):1320-1334.

14. Kim MG, Shon Y, Kim J, Oh YK. Selective activation of anticancer chemotherapy by cancerassociated fibroblasts in the tumor microenvironment. J Natl Cancer Inst. 2017;109(1):djw186.

15. Aertgeerts K, et al. Structural and kinetic analysis of the substrate specificity of human fibroblast activation protein alpha. J Biol Chem. 2005;280(20):19441-19444.

16. LeBeau AM, Brennen WN, Aggarwal S, Denmeade SR. Targeting the cancer stroma with a fibroblast activation protein-activated promelittin protoxin. Mol Cancer Ther. 2009;8(5):1378-1386.

17. Owellen RJ, Hartke CA, Dickerson RM, Hains FO. Inhibition of tubulin-microtubule polymerization by drugs of the Vinca alkaloid class. Cancer Res. 1976;36(4):1499-1502.

18. Poplawski SE, et al. Identification of selective and potent inhibitors of fibroblast activation protein and prolyl oligopeptidase. JMed Chem. 2013;56(9):3467-3477.

19. Wang XM, Yu DM, McCaughan GW, Gorrell MD. Fibroblast activation protein increases apoptosis, cell adhesion, and migration by the LX-2 human stellate cell line. Hepatology. 2005;42(4):935-945

20. Meadows SA, et al. Ala657 and conserved active site residues promote fibroblast activation protein endopeptidase activity via distinct mechanisms of transition state stabilization. Biochemistry. 2007;46(15):4598-4605.

21. Connolly BA, et al. Dipeptide boronic acid inhibitors of dipeptidyl peptidase IV: determinants of potency and in vivo efficacy and safety. J Med Chem. 2008;51(19):6005-6013.
22. Ceelen $\mathrm{W}$, et al. Noninvasive monitoring of radiotherapy-induced microvascular changes using dynamic contrast enhanced magnetic resonance imaging (DCE-MRI) in a colorectal tumor model. Int J Radiat Oncol Biol Phys. 2006;64(4):1188-1196.

23. Mason RP, Zhao D, Liu L, Trawick ML, Pinney KG. A perspective on vascular disrupting agents that interact with tubulin: preclinical tumor imaging and biological assessment. Integr Biol (Camb). 2011;3(4):375-387.

24. Kim KW, et al. Vascular disrupting effect of CKD516: preclinical study using DCE-MRI. Invest New Drugs. 2013;31(5):1097-1106.

25. Thoeny HC, Ross BD. Predicting and monitoring cancer treatment response with diffusionweighted MRI. JMagn Reson Imaging. 2010;32(1):2-16.

26. Schreiber H, Rowley DA. Cancer. Awakening immunity. Science. 2010;330(6005):761-762.

27. Cass CE, Turner AR, Selner M, Allalunis MJ, Tan TH. Effect of lithium on the myelosuppressive and chemotherapeutic activities of vinblastine. Cancer Res. 1981;41(3):1000-1005.

28. Nathanson L, Kaufman SD, Carey RW. Vinblastine, infusion, bleomycin, and cis-dichlorodiammine-platinum chemotherapy in metastatic melanoma. Cancer. 1981;48(6):1290-1294.

29. Valdivieso M, Richman S, Burgess AM, Bodey GP, Freireich EJ. Initial clinical studies of vindesine. Cancer Treat Rep. 1981;65(9-10):873-875.

30. Welford AF, et al. TIE2-expressing macrophages limit the therapeutic efficacy of the vasculardisrupting agent combretastatin A4 phosphate in mice. JClin Invest. 2011;121(5):1969-1973.

31. Shaked Y, et al. Therapy-induced acute recruitment of circulating endothelial progenitor cells to tumors. Science. 2006;313(5794):1785-1787.

32. Taylor M, et al. Reversing resistance to vasculardisrupting agents by blocking late mobilization of circulating endothelial progenitor cells. Cancer Discov. 2012;2(5):434-449.

33. McKeage MJ, Baguley BC. Disrupting established tumor blood vessels: an emerging therapeutic strategy for cancer. Cancer. 2010;116(8):1859-1871.

34. Raza A, Franklin MJ, Dudek AZ. Pericytes and vessel maturation during tumor angiogenesis and metastasis. Am J Hematol. 2010;85(8):593-598.

35. Tonino P, Abreu C. Microvessel density is associated with VEGF and $\alpha$-SMA expression in different regions of human gastrointestinal carcinomas. Cancers (Basel). 2011;3(3):3405-3418.

36. Franco M, Roswall P, Cortez E, Hanahan D, Pietras K. Pericytes promote endothelial cell survival through induction of autocrine VEGF-A signaling and Bcl-w expression. Blood. 2011;118(10):2906-2917.

37. Guan YY, et al. Selective eradication of tumor vascular pericytes by peptide-conjugated nanoparticles for antiangiogenic therapy of melanoma lung metastasis. Biomaterials. 2014;35(9):3060-3070.

38. Loi M, et al. Combined targeting of perivascular and endothelial tumor cells enhances anti-tumor efficacy of liposomal chemotherapy in neuroblastoma. J Control Release. 2010;145(1):66-73.

39. Lu C, et al. Targeting pericytes with a PDGF-B aptamer in human ovarian carcinoma models. Cancer Biol Ther. 2010;9(3):176-182.

40. Murphy EA, et al. Disruption of angiogenesis and tumor growth with an orally active drug that stabilizes the inactive state of PDGFRbeta/B-RAF. Proc Natl Acad Sci U S A. 2010;107(9):4299-4304.

41. Chute JP, Himburg HA. Imatinib tackles lymphoma via the PDGFR $\beta+$ pericyte. Blood. 2013;121(26):5107-5108.

42. Sugimoto H, Mundel TM, Kieran MW, Kalluri R. Identification of fibroblast heterogeneity in the tumor microenvironment. Cancer Biol Ther. 2006;5(12):1640-1646.

43. Bogenrieder T, et al. Expression and localization of aminopeptidase $\mathrm{A}$, aminopeptidase $\mathrm{N}$, and dipeptidyl peptidase IV in benign and malignant human prostate tissue. Prostate. 1997;33(4):225-232.

44. Franklin WA, Christison WH, Colley M, Montag AG, Stephens JK, Hart CE. In situ distribution of the beta-subunit of platelet-derived growth factor receptor in nonneoplastic tissue and in soft tissue tumors. Cancer Res. 1990;50(19):6344-6348.

45. Xian X, et al. Pericytes limit tumor cell metastasis. JClin Invest. 2006;116(3):642-651.

46. Cooke VG, et al. Pericyte depletion results in hypoxia-associated epithelial-to-mesenchymal transition and metastasis mediated by met signaling pathway. Cancer Cell. 2012;21(1):66-81.

47. Keskin D, et al. Targeting vascular pericytes in hypoxic tumors increases lung metastasis via angiopoietin-2. Cell Rep. 2015;10(7):1066-1081.

48. Yang Y, et al. The PDGF-BB-SOX7 axis-modulated IL-33 in pericytes and stromal cells promotes metastasis through tumour-associated macrophages. Nat Commun. 2016;7:11385.

49. Hosaka K, et al. Pericyte-fibroblast transition promotes tumor growth and metastasis. Proc Natl Acad Sci USA. 2016;113(38):E5618-E5627.

50. Moudi M, Go R, Yien CY, Nazre M. Vinca alkaloids. Int J Prev Med. 2013;4(11):1231-1235.

51. Tentler JJ, et al. Patient-derived tumour xenografts as models for oncology drug development. Nat Rev Clin Oncol. 2012;9(6):338-350.

52. Zhang X, et al. A renewable tissue resource of phenotypically stable, biologically and ethnically diverse, patient-derived human breast cancer xenograft models. Cancer Res. 2013;73(15):4885-4897.

53. Reddy JA, et al. Preclinical evaluation of EC145, a folate-vinca alkaloid conjugate. Cancer Res. 2007;67(9):4434-4442.

54. Le Bihan D. Apparent diffusion coefficient and beyond: what diffusion MR imaging can tell us about tissue structure. Radiology. 2013;268(2):318-322.

55. Sourbron SP, Buckley DL. On the scope and interpretation of the Tofts models for DCE-MRI. Magn Reson Med. 2011;66(3):735-745. 